\title{
Neural stem cell transplantation can ameliorate the phenotype of a mouse model of spinal muscular atrophy
}

\author{
Stefania Corti, ${ }^{1,2}$ Monica Nizzardo,, ${ }^{1}$ Martina Nardini,, ${ }^{1}$ Chiara Donadoni, ${ }^{1}$ Sabrina Salani, ${ }^{1}$ \\ Dario Ronchi, ${ }^{1}$ Francesca Saladino, ${ }^{1}$ Andreina Bordoni, ${ }^{1}$ Francesco Fortunato, ${ }^{1}$ Roberto Del Bo, ${ }^{1}$ \\ Dimitra Papadimitriou, ${ }^{1}$ Federica Locatelli, ${ }^{3}$ Giorgia Menozzi, ${ }^{3}$ Sandra Strazzer, ${ }^{3}$ \\ Nereo Bresolin, ${ }^{1,2,3}$ and Giacomo P. Comi ${ }^{1,2}$
}

\begin{abstract}
1Dino Ferrari Centre, Department of Neurological Sciences, University of Milan and IRCCS Foundation Ospedale Maggiore Policlinico, Mangiagalli and Regina Elena, Milan, Italy. ${ }^{2}$ Centre of Excellence on Neurodegenerative Diseases, University of Milan, Milan, Italy.
\end{abstract} 3IRCCS Eugenio Medea, Bosisio Parini, Lecco, Italy.

\begin{abstract}
Spinal muscular atrophy (SMA), a motor neuron disease (MND) and one of the most common genetic causes of infant mortality, currently has no cure. Patients with SMA exhibit muscle weakness and hypotonia. Stem cell transplantation is a potential therapeutic strategy for SMA and other MNDs. In this study, we isolated spinal cord neural stem cells (NSCs) from mice expressing green fluorescent protein only in motor neurons and assessed their therapeutic effects on the phenotype of SMA mice. Intrathecally grafted NSCs migrated into the parenchyma and generated a small proportion of motor neurons. Treated SMA mice exhibited improved neuromuscular function, increased life span, and improved motor unit pathology. Global gene expression analysis of laser-capture-microdissected motor neurons from treated mice showed that the major effect of NSC transplantation was modification of the SMA phenotype toward the wild-type pattern, including changes in RNA metabolism proteins, cell cycle proteins, and actin-binding proteins. NSC transplantation positively affected the SMA disease phenotype, indicating that transplantation of NSCs may be a possible treatment for SMA.
\end{abstract}

\section{Introduction}

Spinal muscular atrophy (SMA) is an autosomal recessive motoneuron disease (MND) and is the second most common genetic disorder leading to death in childhood (1). SMA is caused by deletions or mutations in the telomeric copy of the survival motor neuron 1 gene (SMN1) (2), leading to the depletion of survival motor neuron (SMN) protein levels (3). No effective therapy is currently available for SMA, and treatment is usually supportive. Mice lacking the mouse Smn gene but with 2 copies of the human SMN2 gene and an additional SMN cDNA lacking exon 7 (SMN $\triangle 7)\left(\mathrm{Smn}^{-1}\right.$ $S M N 2^{+/+} S M N \Delta 7^{+/+}$mice; referred to herein as SMA mice) develop a type I SMA phenotype, with a life span of approximately 2 weeks (4). SMA animal models are potentially useful for studying the mechanisms of motoneuron death and provide an in vivo system for testing potential SMA therapies.

Stem cell transplantation could represent a therapeutic approach for MNDs such as SMA. Neural stem cell (NSC) transplantation could improve the neurodegenerative phenotypes through multiple mechanisms complementing neuronal replacement, including the delivery of neuroprotective factors produced by the stem cells (5), the reduction of toxic substances in the microenvironment

Nonstandard abbreviations used: ALDH, aldehyde dehydrogenase; ALS, amyotrophic lateral sclerosis; BDNF, brain-derived neurotrophic factor; CSF, cerebrospinal fluid; ChAT, choline acetyltransferase; GDNF, glial cell line-derived neurotrophic factor; GFAP, glial fibrillary acidic protein; LCM, laser-capture microdissection/microdissected; MAP2, microtubule-associated protein 2; MCP1, monocyte chemoattractant protein-1; MND, motoneuron disease; NF, neurofilament; NMJ, neuromuscular junction; NSC, neural stem cell; NT3, neurotrophin-3; PMN, primary spinal motoneuron; SSC, side scatter; SMA, spinal muscular atrophy; SMN, survival motor neuron; $\mathrm{SMN} \Delta 7$, SMN cDNA lacking exon 7; TA, tibialis anterior.

Conflict of interest: The authors have declared that no conflict of interest exists. Citation for this article: J. Clin. Invest. 118:3316-3330 (2008). doi:10.1172/JCI35432.
(6), and the replacement of other, non-neuronal cells (7). However, the precise identity of these mechanisms has remained an open question. Transplantation of NSCs in SOD1G93A mice, an animal model of amyotrophic lateral sclerosis (ALS), modifies disease progression through both neurogenesis and growth factor release (8). We have previously described the isolation of a subset of selfrenewing, multipotent NSCs on the basis of their aldehyde dehydrogenase (ALDH) activity, characterized as ALDH ${ }^{\text {hi }}$ side scatter ${ }^{\text {lo }}$ $\left(\mathrm{ALDH}^{\text {hisSC }} \mathrm{SS}^{\mathrm{lo}}\right)$ cells. When intrathecally transplanted into $n m d$ mice, an animal model of SMA with respiratory distress type 1 (SMARD1), these cells generate motoneurons that are properly localized in the spinal cord ventral horns. Transplanted $n m d$ animals presented delayed disease progression, sparing of motoneurons and ventral root axons, and increased life span (9).

To our knowledge, stem cell transplantation has never been tested in the SMA model as a potential therapeutic strategy. To determine the therapeutic potential of NSC effects on the SMA phenotype, we intrathecally transplanted ALDH ${ }^{\text {hi SSC }}{ }^{\text {lo }}$ cells into SMA mice. These experiments demonstrate that: (a) ALDH ${ }^{\text {hiSSClo NSCs }}$ can migrate into the parenchyma and generate a small amount of motoneurons; (b) NSC transplantation improves motor unit integrity and the motor function and survival of SMA mice; and (c) after transplantation, the gene expression pattern of endogenous motoneurons was modified toward the wild-type profile. Our data provide evidence that NSC transplantation has a positive effect on the SMA disease phenotype.

\section{Results}

ALDH ${ }^{\text {hiSSClo }}$ NSCs prolong the life and improve the disease phenotype of SMA mice. To examine the ability of NSCs to modify the disease progression of SMA mice, we used a population of these 

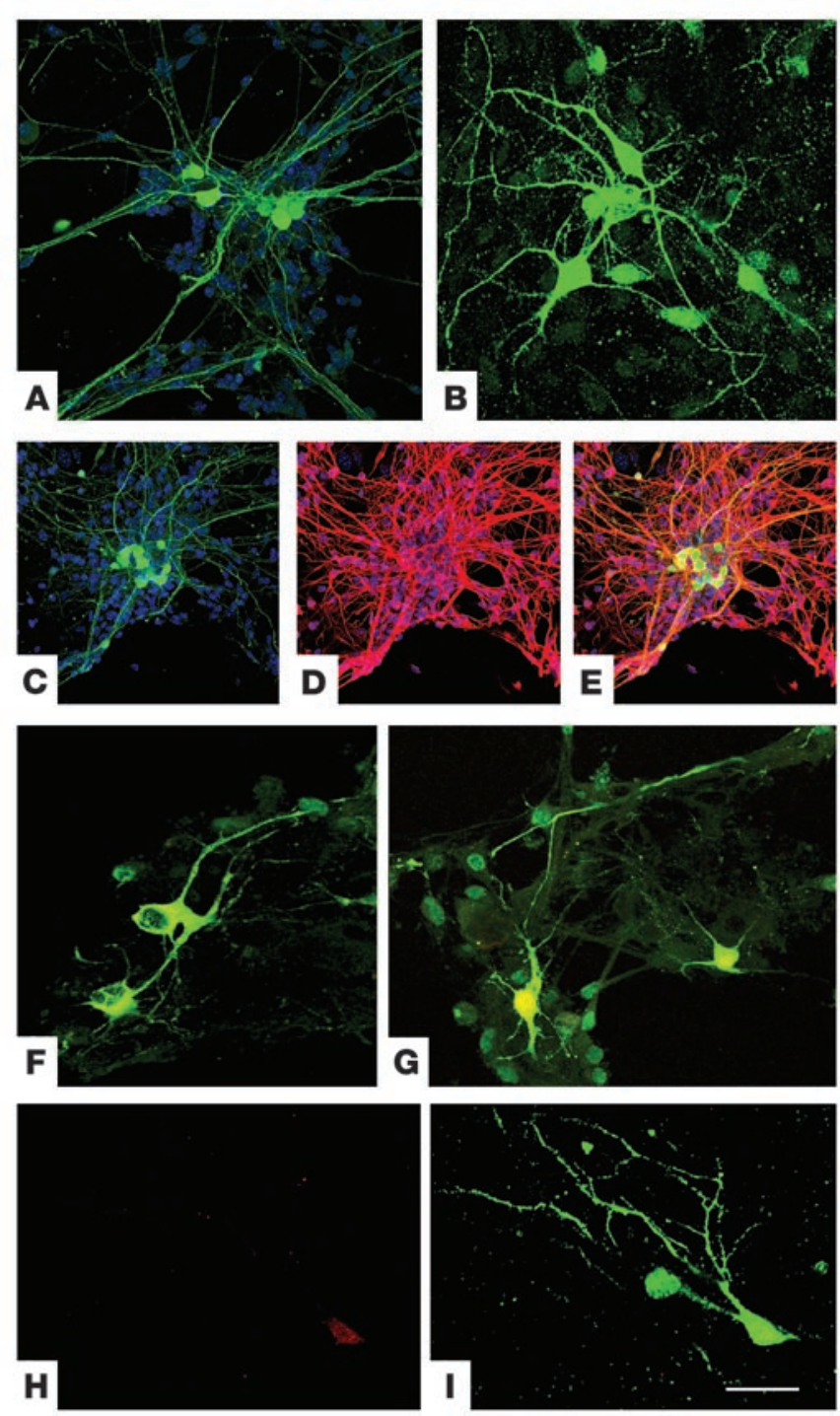

cells from fetal murine spinal cord neurospheres, enriched by FACS based on high ALDH activity and low orthogonal light scattering properties (ALDH ${ }^{\text {hisSC }}{ }^{\text {lo }}$ ), as described previously (9) (Supplemental Figure 1; supplemental material available online with this article; doi:10.1172/JCI35432DS1). These cells are self-renewing and multipotent and can differentiate into the 3 major lineages (9). Indeed, they can differentiate into motoneurons when grown in the presence of retinoic acid (RA) with sonic hedgehog (Shh), after a period of "priming" in culture with specific growth factors $(8,9)$ (Figure 1 ).

After priming the cells in culture for 5 days $(8,9)$, we intrathecally transplanted 20,000 ALDH $^{\text {hiSSClo }}{ }^{\text {lo }}$ cells into SMA mice at 1 day of age (P1). The cell concentration and transplantation protocol were established on the basis of previous results obtained by transplanting $\mathrm{ALDH}^{\text {hiSSClo }}{ }^{\text {lo }}$ into $n m d$ mice (9). Untreated transgenic SMA littermates of treated mice that received only vehicle and $S m n^{+/+} S M N 2^{+/+} S M N \Delta 7^{+/+}$(wild-type for the SMN locus; referred to herein as WT mice) were used as controls. The study was designed so that siblings were distributed equally throughout the treated and control groups and were equally divided between male and female. To identify and monitor directly motoneurons

\section{Figure 1}

$\mathrm{ALDH}^{\text {hiSSClo }}{ }^{\text {lo }}$ cells can differentiate into motoneurons in vitro. After in vitro differentiation, ALDH ${ }^{\text {hiSSClo }}$ cells derived from HB9-GFP transgenic mice that express GFP protein (green) only in motoneurons give rise to neurons with a complex morphology ( $\mathbf{A}$ and $\mathbf{B})$, showing a neuronal and motoneuronal phenotype confirmed by the expression of neuronal marker TuJ1 (C, D, and merged image in E), ChAT (merged images for HB9-GFP and ChAT; F and G), and islet-1 (H and I, merge). Nuclei are stained with DAPI. Scale bars: $70 \mu \mathrm{m}$ (A); $50 \mu \mathrm{m}$ (B and F-I); $100 \mu \mathrm{m}$ (C-E).

derived from $\mathrm{ALDH}^{\text {hisSC }}{ }^{\text {lo }}$ cells, we used transgenic mice expressing GFP under the control of the HB9 promoter in motoneurons (10) as cell donors, thus enabling identification of engrafted motoneurons in the host spinal cord (Figure 2).

When $\mathrm{ALDH}^{\text {hisSClo }}{ }^{\text {lo }}$ NCs were transplanted into SMA mice, the physical appearance of the SMA mice was improved in comparison to that of untreated, age-matched SMA mice (Figure 3A). Treated SMA mice showed significantly improved survival compared with untreated mice (log-rank test; $\chi^{2}=47.97, P<0.00001$; Figure 3B). Mean survival increased from $13.04 \pm 1.73$ days in untreated mice $(n=24)$ to $18.16 \pm 1.78$ days in treated mice $(n=24)$. Survival was extended by 5.12 days, which represented a gain of $39.26 \%$ over the lifetime. The maximum survival was 21 days in treated and 16 days in untreated mice. Figure 3B shows the Kaplan-Meier survival curves for these mice. No statistically significant difference in survival between male and female mice was observed (data not shown).

We also evaluated the survival curve of SMA mice transplanted with undifferentiated $\mathrm{ALDH}^{\text {hiSSC}}{ }^{\text {lo }}$ cells, $\mathrm{ALDH}^{\mathrm{hi}} \mathrm{SSC}^{\mathrm{lo}}$-derived astrocytes, and murine primary fibroblasts (Figure 3B). SMA mice transplanted with primed ALDH ${ }^{\text {hiSSClo }}{ }^{\text {lo }}$ NSCs survived longer than mice grafted with undifferentiated $\mathrm{ALDH}^{\text {hiSSClo }}$ cells, with $\mathrm{ALDH}^{\text {hisSClo }}{ }^{\text {lo }}$ astrocytes, or with primary fibroblasts. The life span of SMA mice transplanted with undifferentiated ALDH ${ }^{\text {hiSSClo }}$ NSCs, although shorter than that observed in mice receiving primed cells, was longer than that of mice receiving astrocytes and fibroblasts (16.92 \pm 1.89 days, $n=24$; primed versus undifferentiated, log-rank test, $\chi^{2}=5.39, P=0.002$; undifferentiated versus astrocytes, log-rank test, $\chi^{2}=13.6, P=0.00023$; undifferentiated versus fibroblasts, $\left.\chi^{2}=28.01, P<0.00001\right)$. Animals grafted with $\mathrm{ALDH}^{\text {hiSSC }}{ }^{\text {lo }}$ NSC-derived astrocytes survived longer than those receiving primary fibroblasts; the survival curve of this latter control group did not differ from that of untreated mice (astrocytes: $14.67 \pm 1.88, n=24$; primed versus astrocytes, log-rank test, $\chi^{2}=26.04, P<0.00001$; astrocytes versus fibroblasts, log-rank test, $\chi^{2}=4.72, P=0.003$; fibroblasts: $13.33 \pm 1.9, n=24$; primed versus fibroblasts, log-rank test, $\chi^{2}=41.97, P<0.00001$; fibroblasts versus vehicle-treated mice, log-rank test, $\chi^{2}=0.76, P=0.38$ ).

One of the first clinical symptoms of the disease is body weight reduction. Untreated SMA mice showed significant weight differences by 5 days of age compared with WT mice $(2.21 \pm 0.18 \mathrm{~g}$ versus $3.62 \pm 0.42 \mathrm{~g}$; ANOVA/Tukey; $P<0.00001 ; n=24$ per group; Figure $3 \mathrm{C})$. The size difference between untreated SMA mice and WT littermates remained evident throughout life. Treated mice showed a greater increase in body weight compared with untreated SMA mice. The growth curve was significantly different at 10-13 days of age (ANOVA/Tukey; $P<0.00001$ ) (Figure 3C).

Neuromuscular evaluation of transplanted animals. Untreated SMA mice presented neuromuscular difficulties at 5 days of age, particularly in righting themselves when placed on their backs. In the 


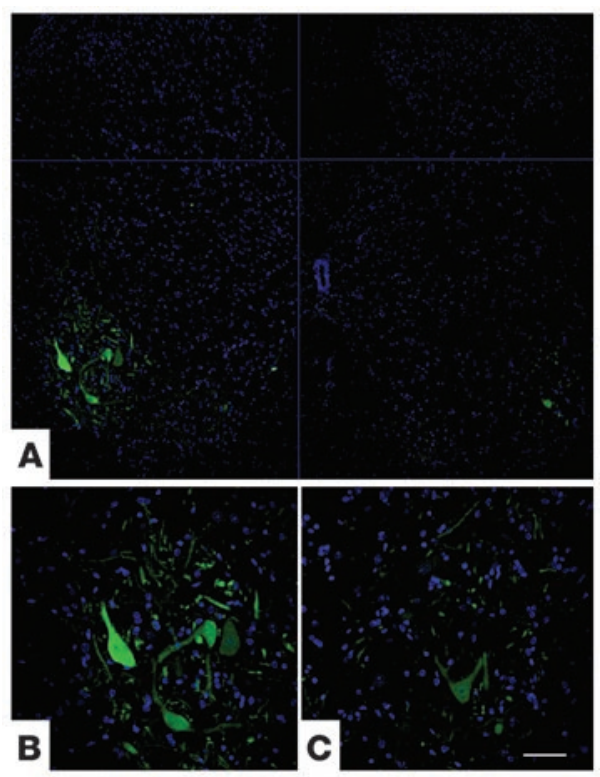

following week of life, this weakness worsened progressively. By 13 days of age, they showed serious difficulty in walking, with frequent falls. When standing on all 4 limbs, they exhibited tremor in the hind limbs. By contrast, at 13 days of age, treated animals showed movement fluidity and gait security (Supplemental Videos 1-3).

To evaluate whether NSC transplantation could provide functional improvement, we tested treated and control mice in the grip assay and open-field tests. These tests have been used previously for neuromuscular assessment in SMA mice of the same age (11). In the grip assay, the average time that control mice could support their weight by forelimb strength increased regularly and proportionately with age (Figure 3D). At 12 days of age, WT mice gripped the metal rail for 10 seconds, but the untreated SMA mice were unable to grip. Conversely, treated mice managed to grip for 3 seconds. At 13 days of age, treated mice presented a stable performance in the grip assay (Supplemental Figure 2), and the grip time of WT mice had increased to 15 seconds (at 13 days, treated versus untreated, ANOVA/Tukey; $P<0.00001$ ).

Observation of the spontaneous activity of mice in an open field represented the second method of functional evaluation (Supplemental Figure 2). At 13 days of age, untreated SMA mice showed severely impaired locomotor activity and exploratory behavior, with compromised surface righting responses. The treated SMA mice presented a reduced number of crossings compared with WT (treated versus WT mice, ANOVA/Tukey; $P<0.00001$ ) but maintained locomotor activity and exhibited some exploration behavior, as indicated by the number of crossings (treated versus untreated mice, ANOVA/Tukey; $P<0.00001)$.

$A L D H^{b i S S C l o} N S C s$ engraft widely and can generate neurons and motoneurons in the SMA parenchyma. To explore the potential mechanisms mediating the beneficial effects of NSC transplantation, we first examined whether ALDH ${ }^{\text {hiSSC }}{ }^{\text {lo }}$ NSCs can contribute by replacing the endogenous dysfunctional mutant cells with a sufficient number of wild-type cells. To address this question, we examined the engraftment distribution of donor cells in the parenchyma and their differentiation fate in the SMA spinal cord. The analysis of cell migration is described in Supplemental Methods and Supplemental Figure 3.

\section{Figure 2}

$\mathrm{ALDH}^{\text {hiSSClo }}$ cells engraft into the ventral horns of SMA mice. (A) Transverse sections of lumbar spinal cord, showing engraftment of transplanted HB9-GFP NSCs after intrathecal injection of P1 mice. ALDH ${ }^{\text {hiSSClo }}$ cells differentiate into motoneuron-like cells that express the HB9-GFP transgene. (B and C) Higher-magnification images (bottom-left and bottom-right sections in $\mathbf{A}$, respectively) of donor-derived HB9-GFP motoneuron-like cells localized in the anterior horns. Scale bar: $200 \mu \mathrm{m}(\mathbf{A}) ; 75 \mu \mathrm{m}$ (B and $\mathbf{C})$.

At the end stage of the disease, we detected transplant-derived motoneurons (HB9-GFP-positive) within the gray matter of the recipient spinal cord (Figure 4A). GFP-positive cells were observed within both the cervical and lumbar enlargement, indicating that NSCs can appropriately acquire a neuronal fate after migration into the parenchyma.

The detected GFP-positive, donor-derived motoneurons appeared morphologically similar to the host motoneurons, albeit with smaller dimensions, which would be expected in newly generated cells. GFP antibody staining distinguished them from the remaining host motoneurons, and the GFP-positive ALDH ${ }^{\text {hiSSC }}{ }^{\text {lo }}$-derived cells extended long processes horizontally into the gray matter.

Unbiased stereological quantification with optical dissectors and random sampling demonstrated that the number of HB9GFP-positive cells was $323 \pm 25$ cells per spinal cord. We also performed FISH analysis for Y chromosome in sex-mismatched transplantation experiments (male cells into female recipients). Unbiased stereological quantification with optical dissectors and random sampling of FISH demonstrated that 2,367 \pm 205 male donor cells were present in the spinal cord parenchyma.

To completely evaluate the phenotype acquired by transplanted cells, we performed confocal immunohistochemical analysis for neuroectodermal antigens combined with FISH analysis for the Y chromosome (Supplemental Figures 4 and 5). Transplanted cells mainly differentiated into neurons, as demonstrated by the double-positive staining for the neuron-specific antigen microtubuleassociated protein 2 (MAP2) and Y chromosome $(41.5 \% \pm 4.7 \%)$. Immunohistochemical analysis for glial antigens (glial fibrillary acidic protein [GFAP] and O4), combined with FISH analysis for Y chromosome, showed the presence of $27.6 \% \pm 5.6 \%$ astrocytes and $3.1 \% \pm 1.1 \%$ oligodendrocytes in all donor male cells. In addition, $25.4 \% \pm 4.5 \%$ cells displayed the characteristics of NSC/progenitor cells, expressing both nestin and Y chromosome.

To confirm the neuronal identity of GFP-positive cells, we used immunohistochemical analysis for neuronal markers followed by confocal microscopy on spinal cord sections of transplanted animals. ALDH ${ }^{\text {hiSSC }}{ }^{\text {lo }}$ GFP-positive cells located in the spinal cord gray matter were immunoreactive for several neuron-specific antigens including neurofilament (NF), MAP2, and nuclear neuron-specific antigen (NeuN) (Figure 4). The complete evaluation of the phenotype acquired by the transplanted cells is described in Supplemental Methods and Supplemental Figure 4. Markers of blood immune cells (lymphocytes and NK cells) or activated microglia identified no signs of graft immunoreaction (data not shown).

To address the possibility of motoneuron replacement by donor cells, we tested to see whether transplanted cells display a motoneuronal phenotype by evaluating the expression of choline acetyltransferase (ChAT) and HB9. These cells resemble true motoneurons morphologically and are positive for both ChAT and HB9 

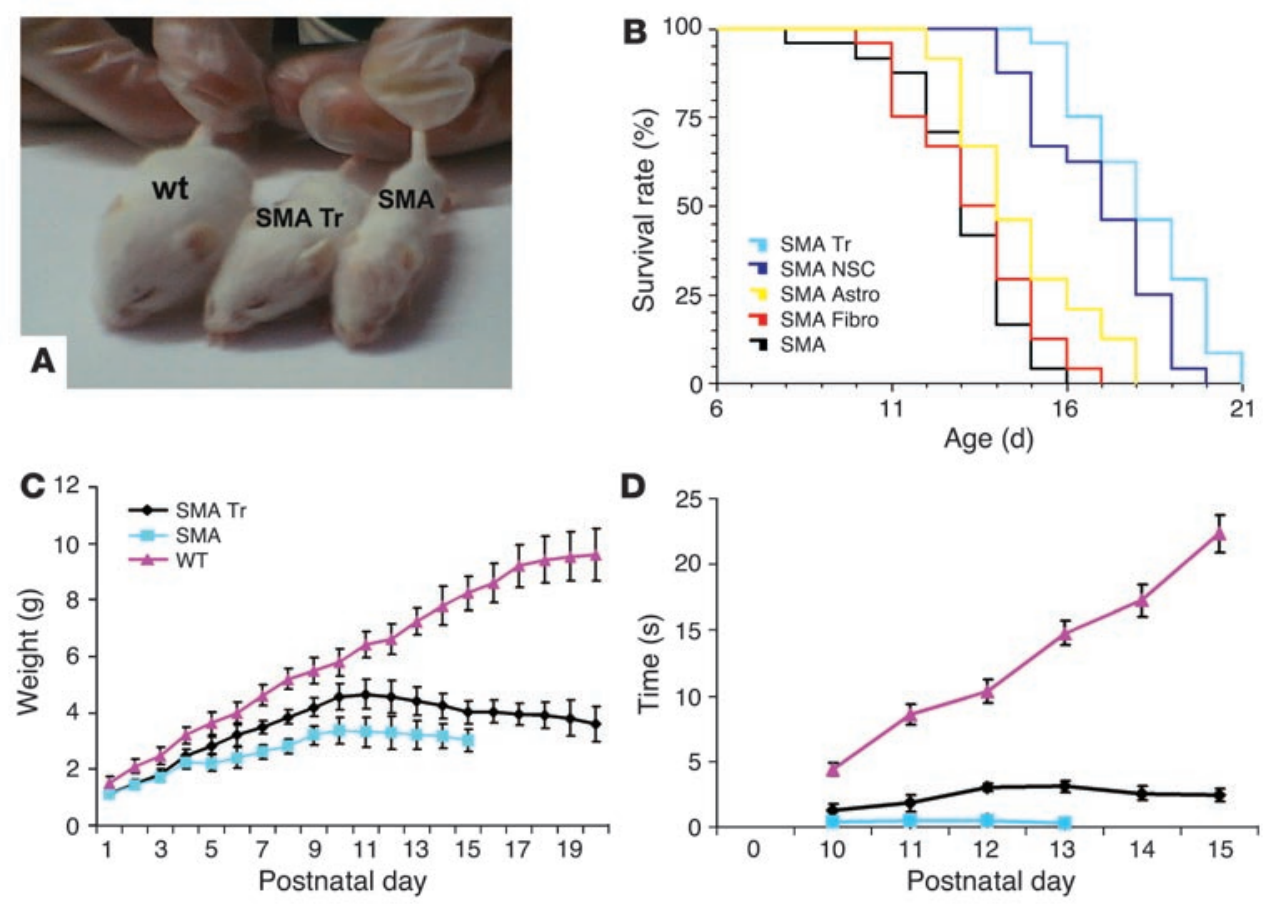

Figure 3

NSC transplantation extends survival, attenuates weight loss, and improves the motor behavior of SMA mice. SMA mice were treated with intrathecal injections of ALDH hiSSClo stem cells or vehicle on day P1. (A) Photographs showing the gross appearance of an NSC-treated SMA mouse (SMA Tr), an untreated SMA mouse (SMA), and a WT mouse. The treated SMA mice were larger than the untreated mice. (B) Kaplan-Meier survival curves of SMA mice treated with "primed" NSCs (SMA Tr), undifferentiated ALDH hiSSClo cells (SMA NSC), ALDH hiSSClo-derived astrocytes (SMA Astro), and primary fibroblasts (SMA Fibro) or untreated mice (SMA; $n=24$ for each group). Survival was significantly extended for mice transplanted with primed NSCs compared with undifferentiated NSCs $(P=0.002$, log-rank test); astrocytes and fibroblasts $(P<0.00001)$; or

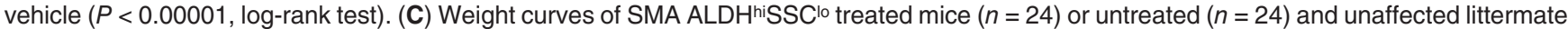
WT controls $(n=24)$. All plots show means of weight at each day, with error bars representing SD. Treated SMA mice displayed an increased growth rate with respect to untreated SMA mice (10-13 days; $P<0.00001)$. (D) Grip time in treated SMA mice $(n=24)$ or untreated SMA $(n=24)$ and unaffected littermate WT controls $(n=24)$. The grip time was statistically different in the untreated and treated SMA mice $(P<0.00001)$ at $12-13$ days of age. Error bars represent SD.

(Figure 4, E-G). According to the stereological count based on ChAT and GFP immunoreactivity, the position within the anterior horns, and the cell dimension (>25 $\mathrm{mm}$ ), we estimated that donor neurons represent $3.5 \%$ of total ChAT motoneurons.

HB9-GFP-derived cells extended their axons horizontally into the white matter, and some cells also extended processes into the ventral roots (a mean of $27 \pm 11$ GFP-positive axons per animal), suggesting that they can elongate their axons toward the periphery (Figure 4, H-J). HB9-GFP axon length was estimated to be $2-3 \mathrm{~mm}$ distal to the root entry into the spinal cord.

Considering the low number of donor motoneurons and their limited axonal extension, we concluded that neuronal replacement was not the main mechanism of the beneficial effect of the NSCs; therefore, we explored further potential mechanisms.

NSC transplantation improves SMA motor unit survival and integrity. To investigate the pathological correlate of this phenotypic amelioration in the SMA mice, we examined a cohort of mice (untreated SMA mice, $n=12$; treated SMA mice, $n=12$; and WT littermates, $n=12$ ) at P10 ( 6 for each condition) and P13 ( 6 for each condition), analyzing muscle and spinal cord tissues.

We evaluated the number and size of motoneurons in the ventral horn of the lumbar spinal cord. At P13 the untreated SMA mice exhibited a substantial motoneuron loss, with a $39.14 \%$ reduction compared with the WT animals $(P<0.00001)$. The mean number of ventral horn neurons per horn per section in WT mice was 9.53, compared with 5.8 in untreated SMA and 7.25 in treated SMA mice (Figure 5E). Counts of spinal cord neurons showed that NSC transplantation conferred significant protection, with only a $23.92 \%$ reduction at 13 days of age $(P<0.00001)$. These neurons had a mean diameter of $36.1 \mu \mathrm{m}$ in WT littermates, $31.2 \mu \mathrm{m}$ in untreated SMA mice, and $34.5 \mu \mathrm{m}$ in treated SMA mice (Figure $5 \mathrm{G})(P<0.00001)$. One of the pathological characteristics of SMA mice is the loss of muscle mass. The SMA myofibers are typically small, but there is no change in the number of nuclei per fiber and no increase in the number of centrally localized nuclei (4). Furthermore, the SMA mice present a reduction of myofibers that may be linked to the loss of function of SMN protein in muscle causing a decline of muscle regenerative capacity (12).

We observed that the mean total cross-sectional area, the diameter of myofibers, and the number of myofibers in the tibialis anterior (TA) muscle were significantly reduced in untreated SMA mice compared with WT littermates (Figure 6). On the other hand, NSC transplantation resulted in a statistically significant increase in total muscle area $(P<0.00001)$, mean myofiber diameter $(P<0.00001$; Figure 6$)$, and mean myofiber number $(P<0.00001)$ with respect to those in untreated mice. 

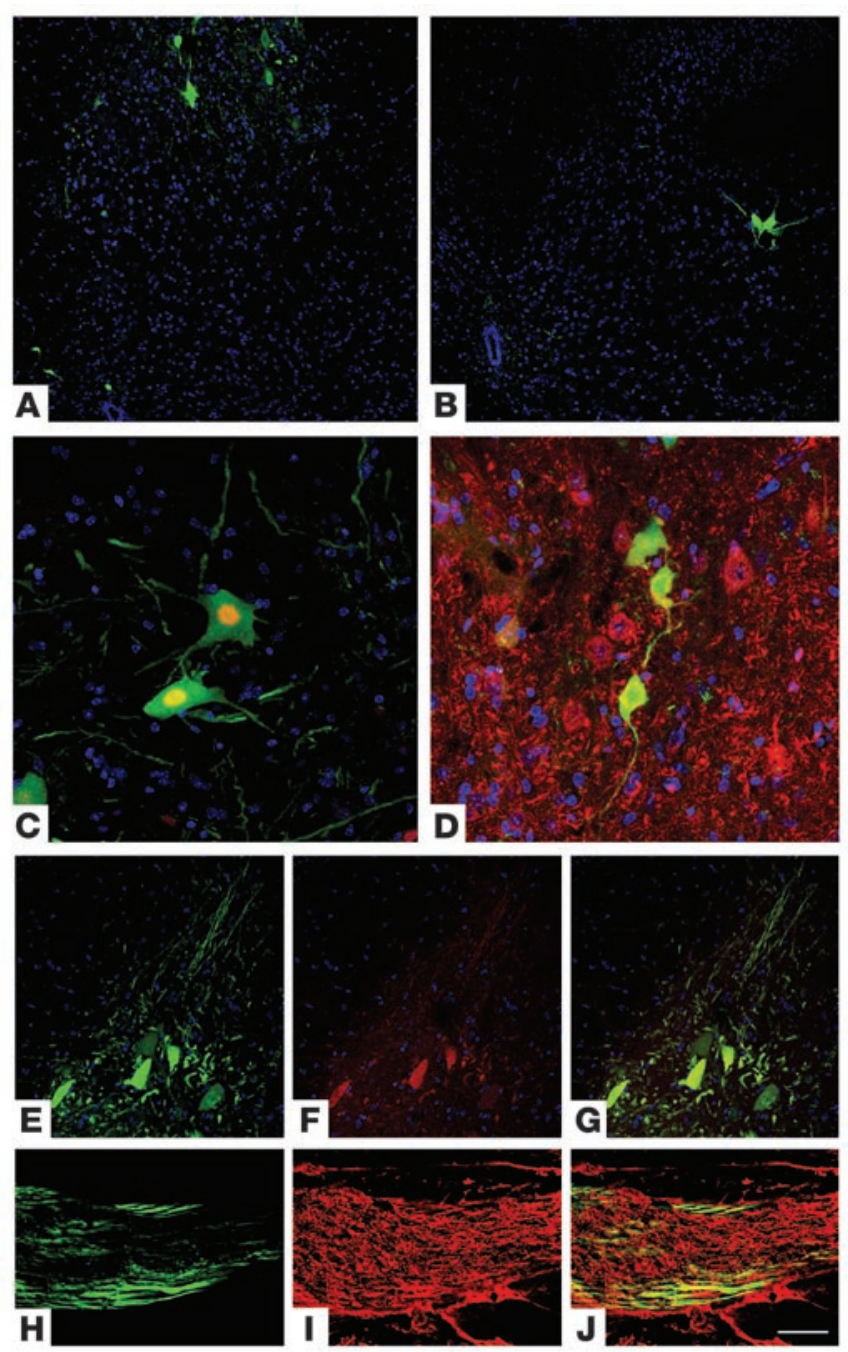

To determine whether NSC transplantation affects motor axon terminals, we performed labeling of neuromuscular junctions (NMJs) on the hind limb muscles of 13-day-old SMA mice treated with NSC or vehicle. The muscle fibers were stained with $\alpha$-bungarotoxin to label the AChR. We found that SMA mice presented a smaller endplate size compared with controls, while the mean NMJ diameter increased in treated compared with untreated mice $(P<0.00001)$ (Figure 6L).

These data also suggest that protecting motor unit integrity is part of the mechanism of improvement seen in treated SMA mice.

NSC transplantation modulates the gene expression profile of SMA mice toward that of WT mice. To gain further insight into these mechanisms of neuroprotection in treated SMA mice, we analyzed the global gene expression profile of isolated motoneurons from treated and untreated SMA and WT mice using laser-capture microdissection (LCM) (Supplemental Figure 6) and microarray analysis. This approach enabled us to analyze changes specific to SMA motoneurons, allowing detection of the effects of interactions with neighboring transplanted cells.

The transcription profiles of motoneurons isolated using LCM from lumbar spinal cords from treated or untreated SMA mice or WT mice were generated using the murine GeneChip Mouse Genome 430A and validated by real-time RT-PCR analyses. The

\section{Figure 4}

ALDH hiSSClo cells transplanted into SMA mice differentiate into motoneurons in vivo. ALDH ${ }^{\text {hiSSClo }}$ cells derived from HB9-GFP mice that express GFP only in motoneurons were transplanted intrathecally. $\mathrm{GFP}^{+}$neurons were detected in the anterior horn of the spinal cord, as shown in spinal cord coronal sections (A, cervical spinal cord; B, lumbar spinal cord). Immunohistochemistry for neuroectodermal markers confirms that these cells are differentiated into neurons. Confocal microscopy showed that GFP+ donor-derived neurons coexpress neuron-specific proteins such as NeuN (C) and MAP2 (D). GFP+ cells present motoneuronal characteristics, as demonstrated by double immunofluorescence staining of GFP (green) under the HB9-specific motoneuronal promoter and cholinergic neurotransmitter (E, GFP; F, ChAT; G, merge). (H-J) HB9-GFP axons (green signal, H) are detected within transplanted SMA ventral roots, labeled with NF (red signal, I), of transplanted SMA mice (J, merge). Scale bar: $250 \mu \mathrm{m}$ (A and B); $75 \mu \mathrm{m}$ (C and D); $100 \mu \mathrm{m}$ (E-G); $50 \mu \mathrm{m}$ (H-J).

endogenous LCM motoneurons were identified by the expression of ChAT and negativity for GFP, the latter being expressed in donor cells only. Differences in gene expression level are presented as ratios of the mean values between untreated SMA and WT mice; treated SMA and WT mice; and treated SMA and untreated SMA mice.

Setting a significant cutoff level to 2.3 -fold change and excluding genes with low expression levels, we detected 42 genes that exhibited varied expression levels in the motoneurons of untreated SMA versus WT mice (Supplemental Table 1) and 31 such genes in the motoneurons of treated versus untreated SMA mice (Supplemental Table 2). With a cutoff level of 1.9-fold change, 165 (motoneurons of untreated SMA versus WT mice) and 137 (motoneurons of treated versus untreated SMA mice) genes showed variable expression levels. The hierarchical cluster analyses clearly discriminated the expression profiles of the 3 experimental conditions (Supplemental Figures 7 and 8).

Interestingly, motoneurons extracted from SMA mice showed a significant increase in the expression of genes involved in the spliceosomal complex and in pre-mRNA splicing and ribosomal RNA processing (Supplemental Table 3). Thirty-four of these genes were upregulated, and 16 were downregulated. These dysregulated genes belong to the RNA helicase DEAD-box family of proteins, the RNA-binding motif proteins, the pre-mRNA processing factor family, the small nuclear ribonucleoprotein family, the heterogeneous nuclear ribonucleoprotein family, and the splicing factor group. Treated mice still showed dysregulation of RNA metabolism-related genes, although with a trend toward the wild-type profile (Supplemental Table 3).

The upregulated genes in SMA motoneurons included those encoding proteins involved in the regulation of transcription, cell cycle control, protein folding (i.e., Hsbp1), and cytoskeletal organization. We detected a 4-fold increase in expression of the cyclindependent kinase inhibitor $1 \mathrm{~A}$ gene $(\mathrm{Cdkn} 1 \mathrm{a})$, which encodes a protein involved in cell growth arrest ( $\mathrm{p} 21)$.

In addition to seeing changes in these gene categories, we observed a reduction in gene expression related to transcription, transport, and actin binding in SMA motoneurons. It has been suggested that the SMN defect causes a reduction in $\beta$-actin protein and mRNA, resulting in reduced axon length and growth cones. We observed a dysregulation of genes coding for proteins involved in actin binding: an upregulation of coactosin-like 1, villin 2, and thymosin 10 beta and downregulation of anillin and solute carrier family 13 member 3 . 
SMA

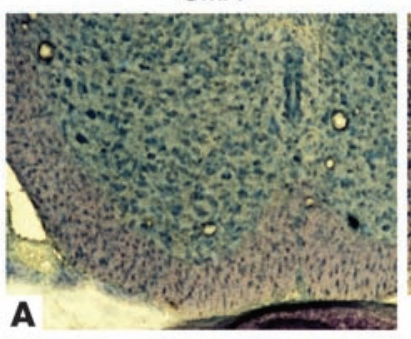

D

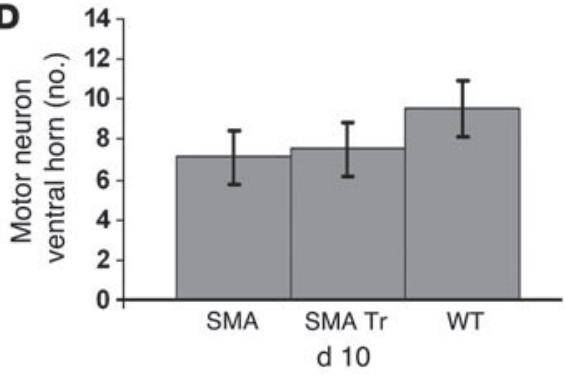

F

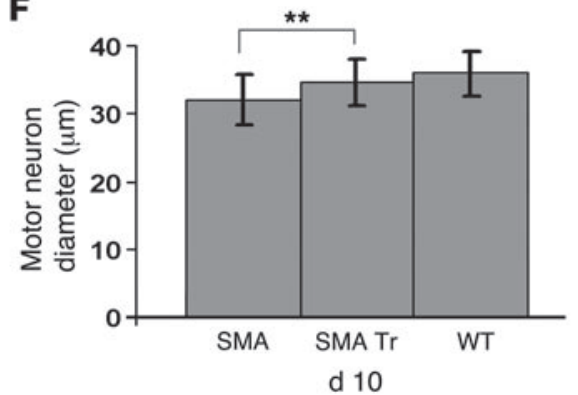

SMA Tr

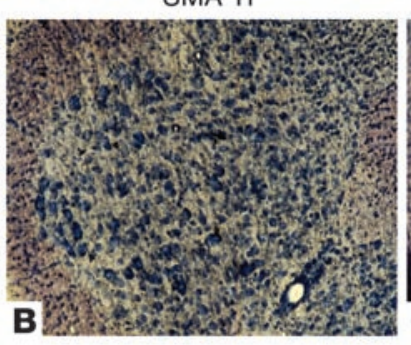

E

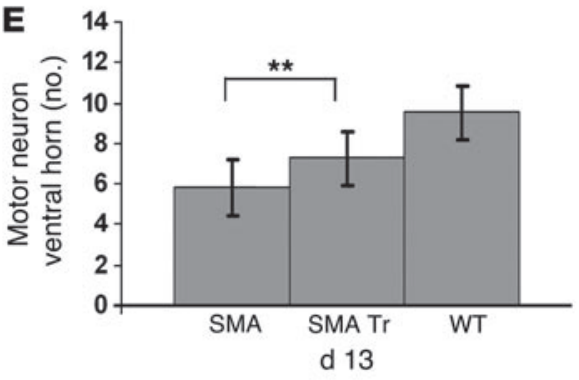

G

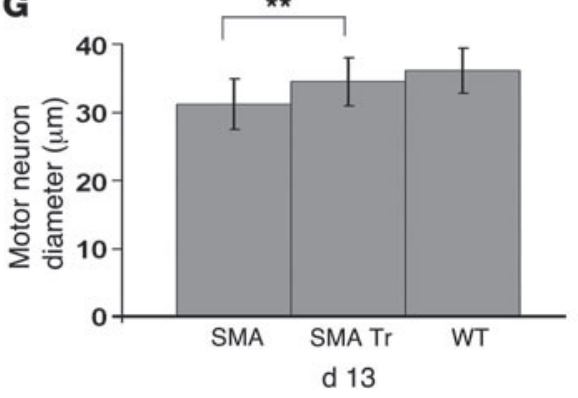

Figure 5

ALDH ${ }^{\text {hiSSClo }}$ cell transplantation increases motoneuron size and number. SMA mice were treated with vehicle $(n=6)$ or NSCs $(n=6)$; WT littermates were treated with vehicle $(n=6)$ on day P1. (A-C) Nissl-stained cross sections of lumbar spinal cord of untreated SMA (A), treated SMA (B), and WT (C) mice. ( $\mathbf{D}$ and $\mathbf{E}$ ) Mean motoneuron number in SMA mice is lower than that in WT littermates and was significantly increased by NSC transplantation $\left({ }^{* *} P<0.00001\right.$, treated versus untreated at 13 days). ( $\mathbf{F}$ and $\mathbf{G}$ ) Mean ventral horn neuron size is smaller in SMA compared with WT mice and was increased by NSC transplantation $\left({ }^{* *} P<0.00001\right)$. Data represent mean motoneuron number and size values \pm SD at $P 10$ ( $D$ and $\mathbf{F}$ ) and P13 (E and G). Scale bar: $300 \mu \mathrm{m}$.
By comparing the profiles of treated and untreated SMA motoneurons, we observed a trend toward the WT profile after NSC transplantation, not only in genes related to RNA metabolism but also in other genes (Supplemental Table 2). However, a subset of genes was differentially expressed in treated compared with untreated SMA mice, with differences unrelated to the WT pattern. These genes belong to a family of "early response genes" and might represent a motoneuron response to the exposure of growth factors released by NSCs (Supplemental Table 2).

To verify the validity of the gene expression levels detected by microarray analysis, we performed quantitative real-time RT-PCR analysis on some genes of interest, which demonstrated mean fold changes in expression levels that were directionally similar to those determined by microarray analysis. It was confirmed that expression of $H s b p 1$ and Cdkn1a was significantly increased in SMA compared with WT mice (untreated SMA mice, Hsbp1: 6.94-fold, $t$ test, $P=0.0002$; Cdkn1a: 8.8-fold, $P=0.014$ ); however, levels in the treated SMA mice showed a tendency toward those in the WT mice (treated SMA mice, Hsbp1: 2.89-fold, treated versus WT, $P=0.02$; Cdkn1a: 5.8-fold, $P=0.003$ ) (Figure 7). Among the downregulated genes, $A n l n$ was decreased, but not significantly (untreated SMA: 0.32-fold, treated SMA: 0.42-fold; untreated SMA versus WT, $t$ test, $P=0.14$; treated SMA versus WT, $P=0.45$ ). Among the genes that were upregulated in treated versus untreated SMA mice, we confirmed increased Dusp1 expression (treated SMA: 8.42-fold; treated versus untreated SMA, $P=0.02$; treated SMA versus WT, $P=0.0007$ ), while Socs 3 showed a trend toward upregulation, although it was not significant (treated SMA: 4.63-fold; treated versus untreated SMA, $P=0.17$; treated SMA versus WT, $t$ test, $P=0.16$ ). Egr 1 expression was significantly upregulated in both treated and untreated SMA mice compared with WT mice, with higher levels in the treated animals (treated SMA: 10.51-fold; untreated SMA: 4.27-fold; untreated SMA versus WT, $P=0.004$; treated SMA versus WT, $P=0.02$; treated versus untreated SMA, $P=0.07$ ) (Figure 7). SMN transcript analysis and Western blot analysis of $\mathrm{p} 21$ and Hsbp1 from SMA primary motoneurons in culture are described in Supplemental Methods and in Supplemental Figure 9.

In vitro analysis of the effect if $A L D H^{\text {hiSSC }} C^{l o}$ NSCs on SMA motoneurons. To further investigate the effect of ALDH ${ }^{\text {hiSSC }}{ }^{\text {lo }}$ NSCs on neurodegeneration in the mutant SMA mouse motoneurons, we used cocultures composed of a bottom layer of primary spinal motoneurons (PMNs) from SMA mice and a top layer of ALDH ${ }^{\text {hiSSClo }}$ NSCs seeded onto a microporous membrane, which permits the diffusion of soluble factors only into the lower compartment.

Because of results indicating that an SMN defect causes reduced axon growth (13), we examined whether NSCs promoted the correction of this phenotype. For measuring neurite length, motoneurons were fixed after 7 days in culture and immunostained with antibodies against the microtubule-associated proteins MAP2 and phosphorylated tau protein (phospho-tau) for identification of dendritic and axonal processes. Mean axon length was significantly shorter (32\%) in SMA motoneurons without coculture with respect to WT $(210.6 \pm 23.1 \mu \mathrm{m}$ versus $310.5 \pm 27.2 \mu \mathrm{m}$, 
SMA

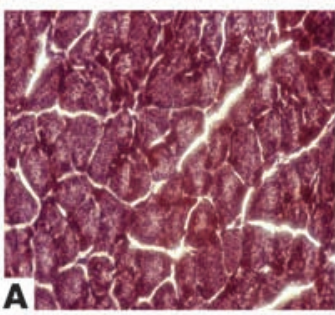

D

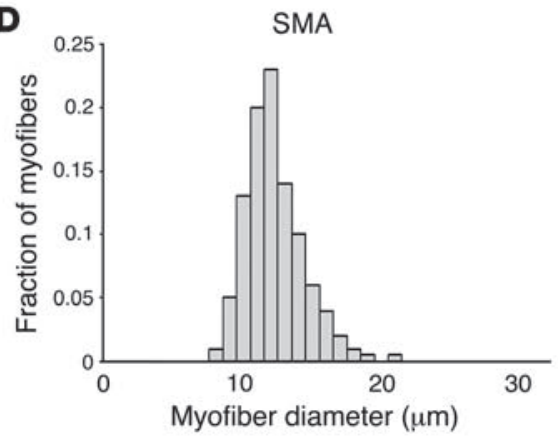

SMA Tr

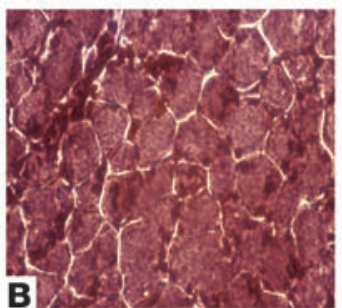

WT

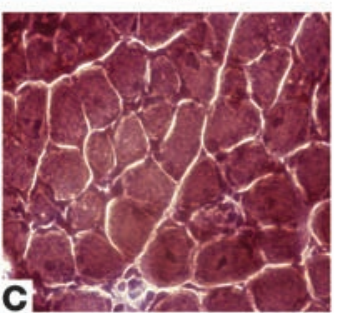

E

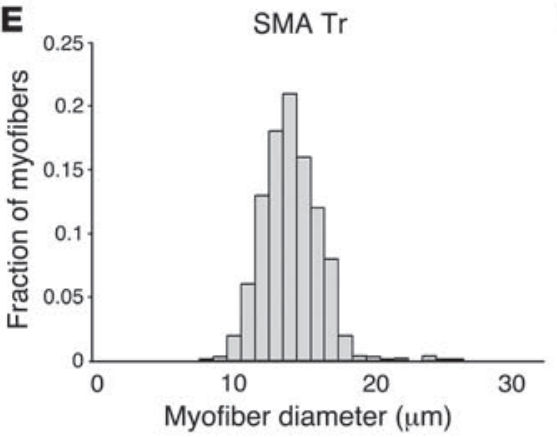

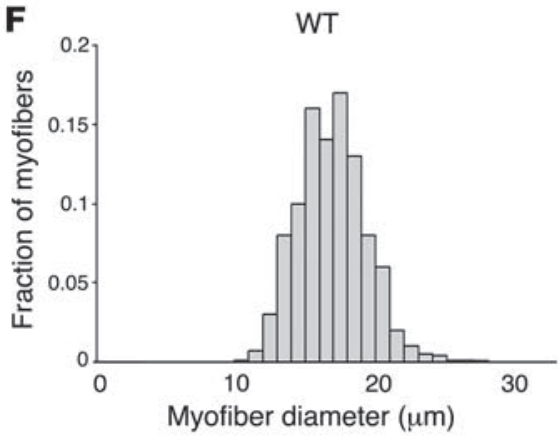
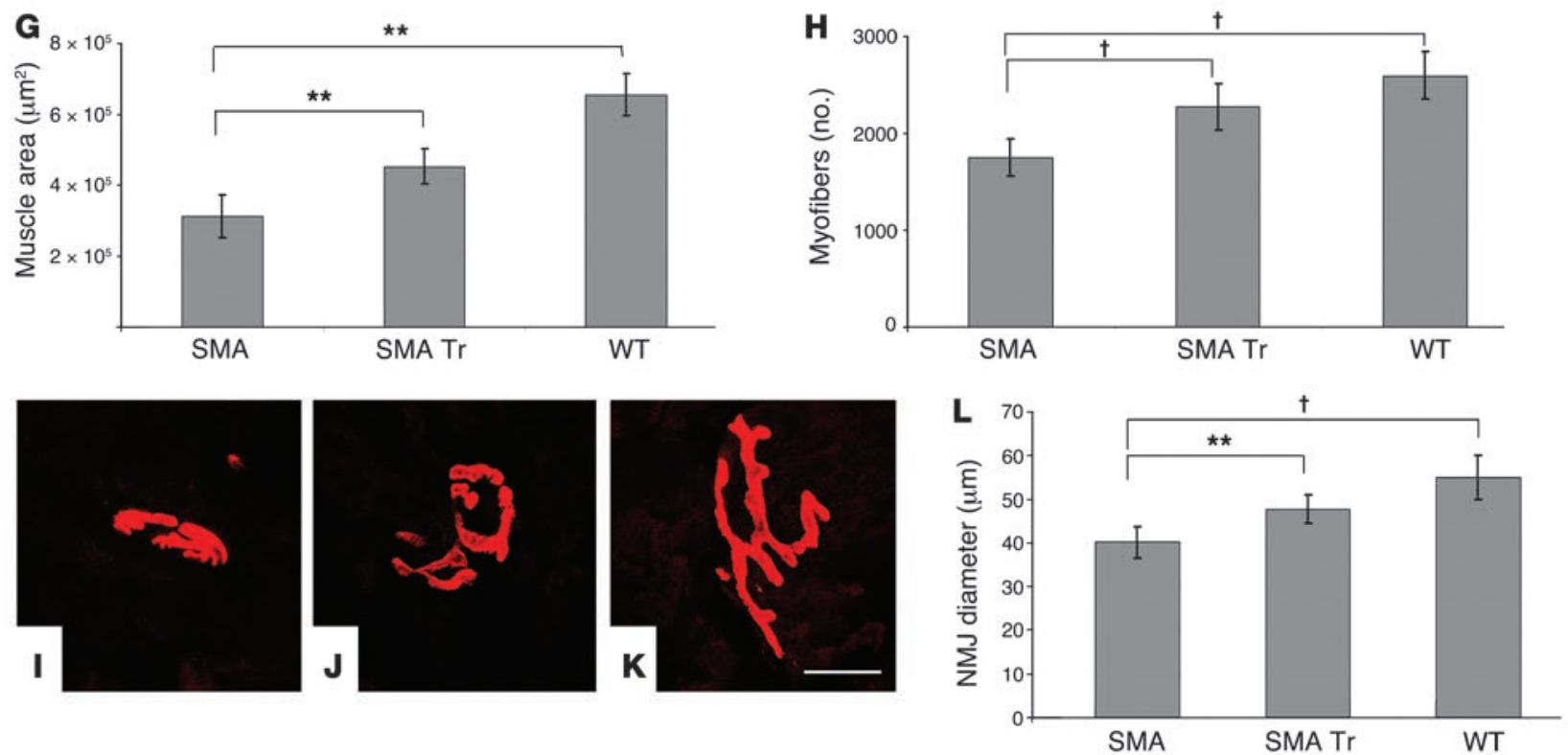

Figure 6

NSC transplantation ameliorates muscle innervation in SMA mice. SMA mice were treated with vehicle $(n=6)$ or NSCs $(n=6)$, and WT littermates were treated with vehicle $(n=6)$ on days P1. (A-C) H\&E-stained cross sections of TA muscle. (D-F) Histograms of myofiber diameters. (G) Mean TA muscle cross-sectional area was reduced in SMA mice compared with WT littermates and increased after NSC transplantation $\left({ }^{\star *} P<0.00001\right)$. Data represent mean values \pm SD. (H) Mean TA muscle total myofiber number was reduced in SMA mice compared with WT littermates and increased after NSC treatment $\left({ }^{\dagger} P<0.00001\right)$. (I-K) Immunofluorescence analysis of NMJs was performed within the gastrocnemii of SMA mice treated with NSCs (I) or vehicle (J) or of WT littermates (P13) (K). $\alpha$-Bungarotoxin was used to label AChR (red). (L) Histogram showing the mean NMJ diameter in treated and untreated SMA and wild-type gastrocnemii at $\mathrm{P} 13$, demonstrating an increased size in treated mice $\left({ }^{* \star} P<0.00001\right.$, treated versus untreated SMA; ${ }^{\dagger} P<0.00001$, SMA versus WT). Scale bar: $300 \mu \mathrm{m}(\mathbf{A}-\mathbf{C}) ; 10 \mu \mathrm{m}(\mathbf{I}-\mathbf{K})$.

$P<0.00001)$, whereas the SMA PMN axons, when cocultured in the presence of NSCs, were significantly longer than the untreated ones $(266.7 \pm 21.1 \mu \mathrm{m}, P<0.00001)$ (Figure 8$)$. We then measured the size of axonal growth cones. Growth cones of SMN-deficient motoneurons were significantly smaller than those of WT; however, after NSC coculture, we observed an increase in size $(P<0.00001$, treated versus untreated SMA mice). Dendrite out- growth was not altered, and neither was the survival of PMNs in treated and untreated SMA compared with WT mice.

Possible mechanisms of effect of primed ALDH $H^{\text {bi SSC }} \mathrm{C}^{\text {lo }}$ NSCs. We sought to define the molecular mechanisms through which primed ALDH ${ }^{\text {hiSSClo }}{ }^{\text {lo }}$ NSCs may ameliorate the SMA phenotype. We used Luminex multi-analyte profiling (xMAP) technology to simultaneously detect and quantify 26 different mouse 
A
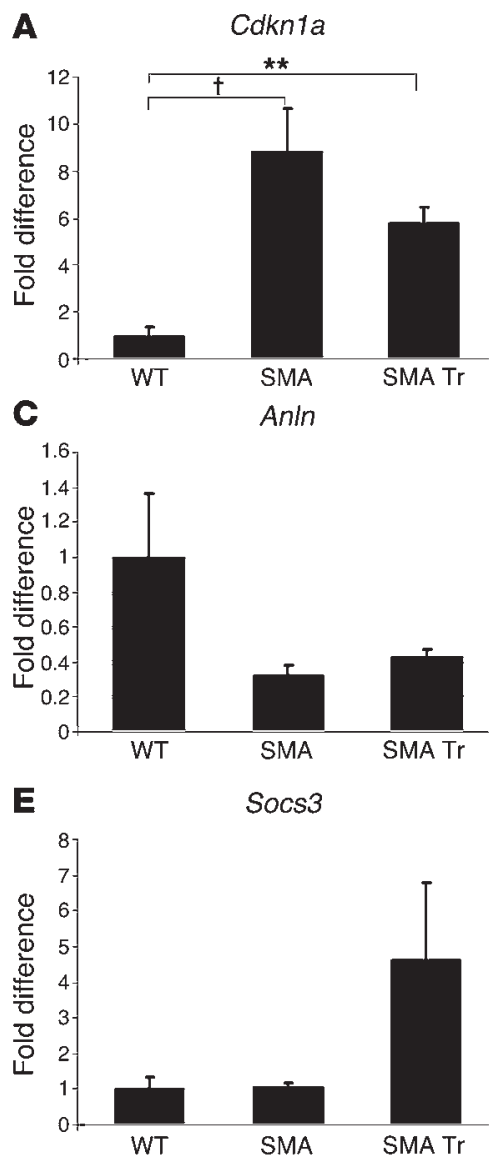

B
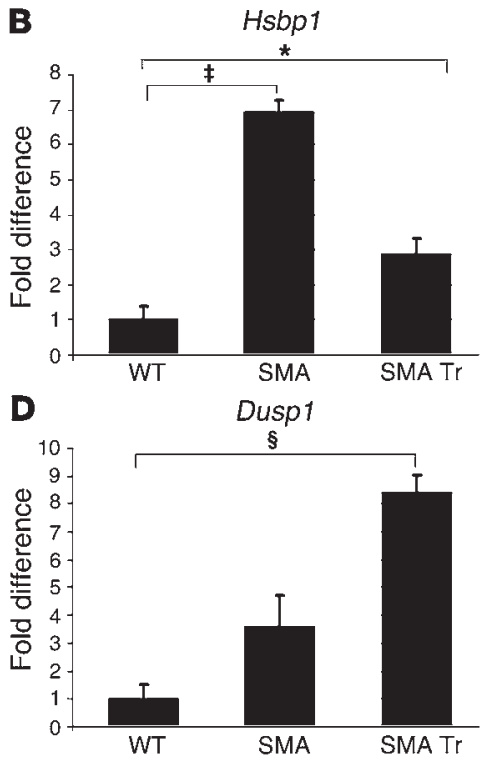

$\mathbf{F}$

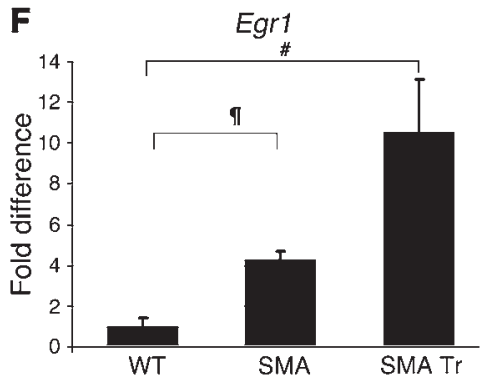

\section{Figure 7}

Real-time PCR analysis of endogenous LCM motoneurons after cell transplantation in SMA mice. We observed a significant upregulation of Cdkn1a (A) and Hsbp1 (B) in SMA compared with WT motoneurons ( $t$ test, ${ }^{\dagger} P=0.0002$ and $\ddagger P=0.014)$; these genes were downregulated after transplantation. ${ }^{\star} P<0.05 ;{ }^{*} P<0.01$. (C) Downregulation of AnIn was detected in SMA motoneurons, although these changes did not reach significance $(P=0.14)$.

(D) We observed upregulation of Dusp1 in treated SMA motoneurons compared with untreated SMA $(P=0.02)$ and WT motoneurons $(\$ P=0.0007)$. (E) Socs3 expression was increased more in treated than in untreated SMA mice, although the difference did not reach significance (treated SMA: $P=0.17$ versus untreated; $P=0.16$ versus $\mathrm{WT})$. (F) Egr1 was significantly upregulated in both treated and untreated SMA mice compared with WT ( $P=0.004$, untreated SMA versus WT; $\# P=0.02$, treated SMA versus WT; $P=0.07$, treated versus untreated SMA). Error bars indicate SD. cytokines in the cell supernatants of primed ALDH ${ }^{\text {hisSC }}{ }^{\text {lo }}$ NSCs, undifferentiated cells, astrocytes, and fibroblasts (Supplemen-

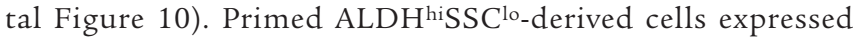
significantly higher levels of VEGF $(1,424.54 \pm 106.62 \mathrm{pg} / \mathrm{ml}$; $P<0.00001)$ compared with the other cell types. Moreover, all

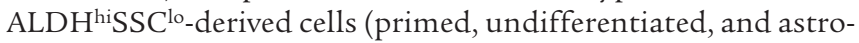
cytes) expressed significantly higher levels of VEGF than did fibroblasts $(P<0.00001)$. ELISA confirmed the differences in VEGF levels as determined by the Multiplex immunoassay (data not shown). Furthermore, primed ALDH ${ }^{\text {hiSSC }}{ }^{\text {lo }}$ NSCs secreted significantly higher quantities of the mouse chemokine $\mathrm{KC}$ (CXCL1) and of G-CSF than did other cell types (KC: primed versus undifferentiated, $P=0.02$; primed versus astrocytes and primed versus fibroblasts, $P<0.00001$; G-CSF: primed versus other cells, $P<0.00001)$. All cell types analyzed expressed high levels of monocyte chemoattractant protein-1 (MCP1), with the highest levels expressed by fibroblasts (MCP1: primed versus undifferentiated, $P=0.000013$; primed versus astrocytes, $P=0.000013$; primed versus fibroblasts, $P=0.00002)$. Other proinflammatory cytokines were either not expressed or were

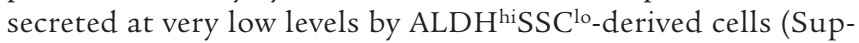
plemental Figure 10).

We also analyzed by ELISA the levels of other growth factors not included in the above-mentioned array to define the profile of neurotrophins: glial cell line-derived neurotrophic factor (GDNF), brain-derived neurotrophic factor (BDNF), neurotrophin-3 (NT3), and TGF- $\alpha$ (Supplemental Figure 11). Primed $\mathrm{ALDH}^{\text {hisSClo }}{ }^{\text {lo }}$ NSCs secreted significant amounts of GDNF
$(731.5 \pm 77.66 \mathrm{pg} / \mathrm{ml}$; primed versus other cells, $P<0.00001)$, BDNF $(501.83 \pm 48.54 \mathrm{pg} / \mathrm{ml}$; primed versus undifferentiated, $P=0.00013$; primed versus astrocytes and primed versus fibroblasts, $P<0.00001$, respectively), TGF- $\alpha(118 \pm 8.37 \mathrm{pg} / \mathrm{ml}$; primed versus other cells, $P<0.00001)$, and NT3 $(156.36 \pm 16.55 \mathrm{pg} / \mathrm{ml}$; primed versus other cells, $P<0.00001)$.

We argued that upregulation of the expression levels of neuro-

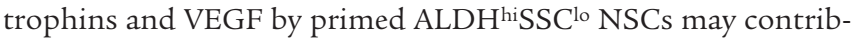
ute to the differential axon length enhancement seen in coculture when we compared primed ALDH ${ }^{\text {hisS }} \mathrm{SC}^{\text {lo }}$ NSCs and SMA PMNs. To test this hypothesis, we neutralized these cytokines (GDNF, BDNF, TGF- $\alpha$, NT3, and VEGF) individually or in combination by adding neutralizing antibodies to the coculture media. Neutralizing antibodies significantly diminished the length of axons of SMA motor neurons (GDNF versus control, $P<0.00001$; BDNF versus control, $P<0.00001$; TGF- $\alpha$ versus control, $P=0.00004$; NT3 versus control, $P<0.00001$; VEGF versus control, $P<0.00001$; Supplemental Figure 11E).

These data suggest that primed ALDH ${ }^{\text {hisSC }}{ }^{\text {lo }}$ cells enhance SMA motor neuron axon length by producing growth factors and suggest that these substances may also account for the axonal protection of SMA host motor neurons seen in transplanted animals in vivo.

\section{Discussion}

SMA is a devastating, untreatable neuromuscular disease caused by reduced expression of the SMN protein leading to a loss of motoneurons in the spinal cord. Stem cell transplantation is a potential therapeutic strategy for SMA, as it results in cell replace- 

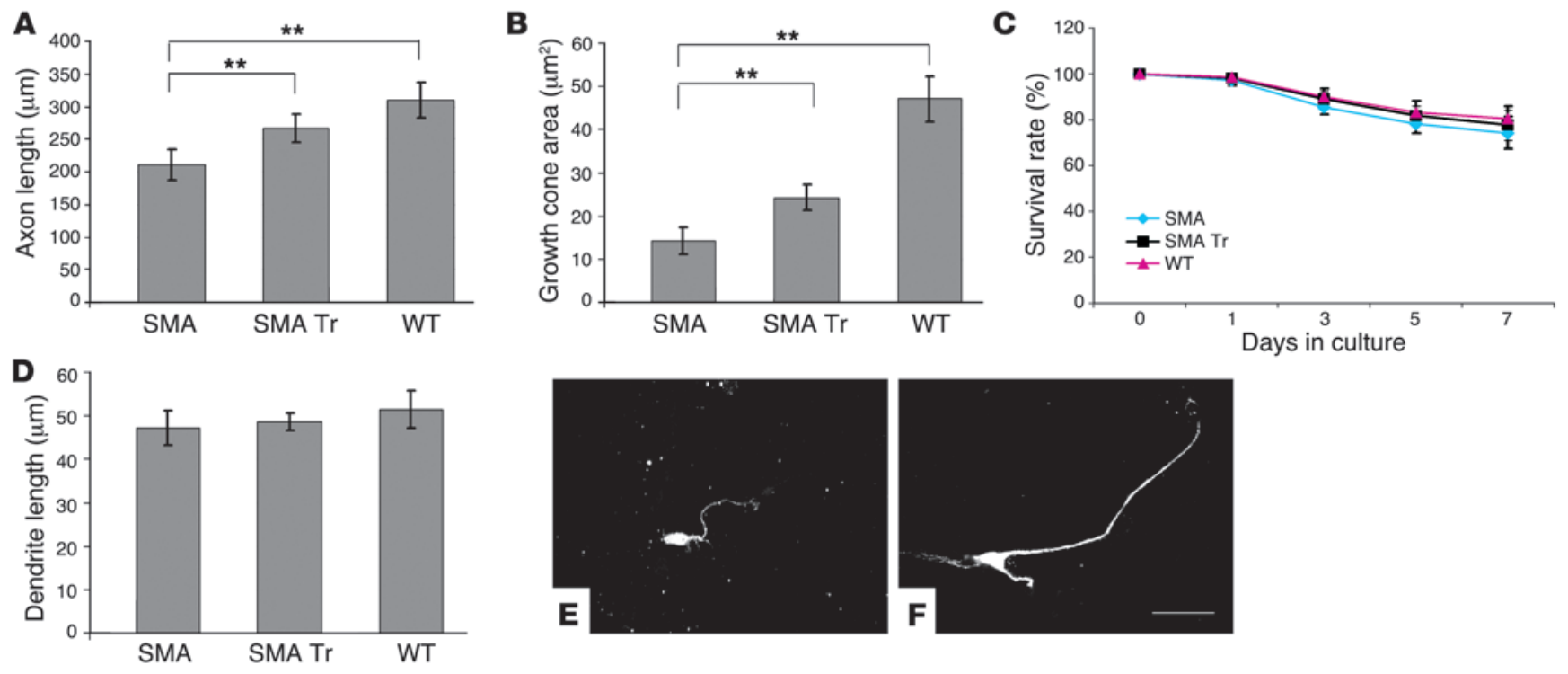

\section{Figure 8}

$\mathrm{ALDH}^{\text {hiSSClo }}$ cells ameliorate the phenotype of primary motoneurons of SMA mice in coculture assay. Average length of axons (A) and growth cone area (B) of motoneurons from both SMA and WT mice, with and without coculture with NSCs. Motoneurons from SMA mice exhibit a significant reduction in axon length and growth cones with respect to WT $\left({ }^{* *} P<0.00001\right)$. Coculture with NSCs significantly improved axon outgrowth of SMA mice $\left({ }^{* *} P<0.00001\right)$. Neither survival $(\mathbf{C})$ nor length of dendritic processes (D) was affected in either treated or untreated SMA mice. $(\mathbf{E}$ and $\mathbf{F})$ Immunostaining of untreated $(\mathbf{E})$ and treated $(\mathbf{F})$ SMA motoneurons with antibodies against axon-specific phospho-tau protein, showing their axon outgrowth. Scale bar: $50 \mu \mathrm{m}$.

ment as well as the activation of molecular and cellular mechanisms that support endogenous neuronal functions and offer protection against degeneration.

We and others have previously demonstrated that NSC transplantation may ameliorate MND phenotypes (8, 9, 14, 15). Here, we demonstrated that $\mathrm{ALDH}^{\text {hiSSClo }}{ }^{\text {lo }}$ stem cells, transplanted into the cerebrospinal fluid (CSF), migrate into the SMA spinal cord, generate motoneuron-like cells, and significantly improve the neurological phenotype and survival of SMA mice. Indeed, cell transplantation ameliorates the SMA pathology and protects endogenous motoneurons. Our results provide what we believe to be the first demonstration that the transplantation of stem cells could have a beneficial role in the course of SMA disease.

NSCs exhibit variable biological properties, depending on their source and culture conditions, because they derive from neurospheres containing heterogeneous cell populations at various stages of differentiation. Consistent with previous results, we found that the isolation of a defined NSC subpopulation is of critical importance for the acquisition of complex neuronal phenotypes such as the motoneuronal phenotype. Various neural stem/progenitor cells may respond differently to instructive cues in the recipient environment. The spinal cord is a non-neurogenic site; however, exposure of donor cells to growth factors and morphogens before transplantation may override the suppressive signals produced by the host environment (16). In fact, the in vitro priming procedure may enable the NSCs to overcome the glial inhibitory signals in the host spinal cord, giving rise both to neurons and cholinergic, motoneuron-like cells.

Intrathecally transplanted NSCs migrated extensively throughout the SMA spinal cord, spreading along both the cervical and lumbar spinal cord tracts. Neuronal death occurring in the SMA spinal cord may represent a possible signal that promotes NSC engraftment and triggers the replication and neurogenesis of exogenous stem cells. ALDH ${ }^{\text {hisSC }}{ }^{\text {lo }}$ cells gave rise to both neurons and motoneurons in vivo after transplantation. Newly generated motoneurons showed the typical morphology and expressed appropriate neuroectodermal proteins and cholinergic neurotransmitters.

Transplanted SMA mice showed an amelioration of the motoneuron phenotype as demonstrated by neuromuscular function tests and increased survival. ALDH ${ }^{\text {hiSSClo }}{ }^{\text {lo }}$ cell transplantation performed at birth significantly prolonged survival, by $39.26 \%$, compared with no treatment. These observations correlated with the neuropathological analysis, which showed a significant reduction in motoneuron loss at 13 days of age as compared with vehicle treatment. Indeed, we demonstrated that the transplantation of primed ALDH ${ }^{\text {hiSSClo }}{ }^{\text {NSCs improves survival }}$ of SMA mice significantly more than graft of other cell types (undifferentiated cells, astrocytes, and fibroblasts), confirming the hypothesis of the specificity of NSC action and the advantage of primed NSCs.

The increased survival time we observed with NSCs, though limited, is relevant considering previously reported results of 2 gene therapy experimental trials using SMA mice. The first of these trials, based on the gene transfer of cardiotrophin-1 through intramuscular injection of adenoviral vectors, induced an extension of the life span in another mouse model of $30 \%$ (17). The second, based on multiple single injections in various muscles of a lentiviral vector expressing $S M N$, restored SMN in motoneurons and increased life expectancy by an average of 3 and 5 days (20\% and $38 \%$ ), compared with LacZ and untreated animals, respectively (18). The difficulties in achieving a significant increase in survival by using different approaches suggest that further understanding 
of the pathogenetic mechanisms of motoneuron death is fundamental to the development of a therapy for SMA.

Among all of the factors that could have contributed to the phenotypic changes, we suggest that the donor-derived motoneurons had the smallest impact on functional recovery. However, the demonstration that motoneurons can be generated from $\mathrm{ALDH}^{\text {hisSC }}{ }^{\text {lo }}$ cells after transplantation with a minimally invasive CSF injection highlights a possible new approach for the replacement of degenerating motoneurons. Furthermore, these data support the usefulness of SMA mice as a tool in the development of CSF transplantation strategies and in the study of exogenous motoneuron generation.

Although NSC transplantation and neurogenesis were not sufficient to rescue fully the SMA phenotype, the behavioral and survival improvement was evident. The neurological amelioration was associated with a significant change in spinal cord pathology, and the motoneuron count in treated compared with untreated mice demonstrated a significant reduction in neural cell death.

Consistent with previous reports (4), we observed a reduction in the number and size of myofibers as well as in the dimensions of endplates in SMA mice compared with heterozygous littermates. Interestingly, the transplanted mice presented ameliorated muscle pathology and increased NMJ size, suggesting improved survival and integrity of the entire motor unit. We believe that these data explain the clinical benefit.

To investigate the molecular events that occurred in SMA motoneurons after cell transplantation, we performed global gene expression profile analysis on isolated endogenous motoneurons. We detected a number of transcripts with significant differential expression in the lumbar spinal cord motoneurons of treated and untreated SMA compared with WT mice. We demonstrated a variation in the level of genes involved in RNA metabolism. This observation is in agreement with the role that SMN plays in RNA processing (19). In addition, it is in line with previous observations regarding the overexpression of genes involved in this process and/or the interactions with SMN in the spinal cord of SMA mice (20). The activation of these genes might represent an adaptive but insufficient response of an RNA processing pathway to the lack of a principal component (20). However, it cannot be excluded that this disruption exerts a pathogenetic effect by modifying the level/ stability of the downstream target RNAs. Furthermore, recent work has shown that SMN deficiency causes tissue-specific perturbations in the pattern of snRNAs and widespread defects in splicing (21).

We and others (20-22) observed an increase in the mRNA and protein levels of 21 in SMA tissues. It has been described that in the absence of SMN, the KH-type splicing regulatory protein is misregulated and that this is correlated with the increased mRNA stability of its mRNA target, $p 21$ (22). Abnormal levels of p21 protein, known to play a role in cell cycle and cell growth arrest, may contribute to the overall SMA pathogenesis; this conclusion is suggested by the presence of myofibers that appear to have undergone arrested development (23) and that express abnormally increased amounts of developmental isoforms of myosin (24).

We also observed upregulation of the Hspb1/HSP27 transcript. Small heat shock proteins (sHSPs) including Hspb1/HSP27 have multiple functions (including chaperone activity, protection against oxidative stress and aging, and interference with apoptosis) and have a neuroprotective role in neurological disorders (25). Consistent with our data, SOD1 mice, an animal model of ALS, presented an increased level of Hsbp1 (26). Protein aggregation plays an important role in ALS and an undetermined role in SMA pathogenesis.
HSPs such as Hsbp1 may modulate these processes. Indeed, some forms of Charcot-Marie-Tooth disease and of distal hereditary motor neuropathy carry mutations in the HSP27 gene (27).

Moreover, we detected a dysregulation of genes encoding proteins with an actin-binding function: an upregulation of coactosin-like 1 , villin 2 , and thymosin 10 beta and a downregulation of anillin and solute carrier family 13 member 3 . The SMN deficit reduced the $\beta$-actin mRNA and protein levels in distal axons, probably leading to alterations in other $\beta$-actin-binding proteins $(13,28)$.

Based on the direct comparison between the treated and untreated SMA motoneuron profiles, we hypothesize that the major effect of NSC transplantation was to modify the SMA phenotype toward the WT pattern, a process involving the RNA metabolism genes, as well as others. On the other hand, a subset of genes found to be differentially expressed in treated compared with untreated SMA mice were not present in the WT pattern. These genes (Dusp1, Socs3, and Egr1) are involved in early response, and their upregulation could be linked to the response of motoneurons to the exposure to growth factors released by NSCs. Indeed, we identified upregulation of SMN full-length transcript in endogenous SMA motoneurons after NSC transplantation. Overall, we believe that the modification of the gene expression profile after treatment explains the amelioration of the SMA motoneuron phenotype, as demonstrated both in vivo and in vitro by the increased axon length and growth cone outgrowth in the functional and survival assays.

We hypothesize that the beneficial effects observed after stem cell transplantation arise from multiple events involving the transplanted cells. Transplanted cells may have a role in functional recovery, serving as "chaperones" for host neurons and providing neuroprotective substances. To address the mechanisms underlying the effects of the transplanted stem cells, we investigated the profile of cytokine and growth factor production. We sought to define molecular factors that may account for the effect of primed ALDH ${ }^{\text {hiSSClo }}{ }^{\text {lo }}$ NSCs on the SMA phenotype. Primed ALDH hiSSClo NSCs differentially secreted 7 soluble factors compared with the other cell types: these factors include VEGF, KC, G-CSF, and several neurotrophins (BDNF, GDNF, NT3, and TGF- $\alpha$ ). We found that these factors individually affected axonal growth of PMNs

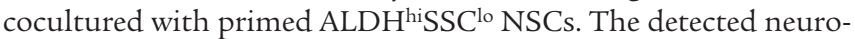
trophins have previously been shown to have a neuroprotective role in motoneurons both in vitro and in vivo (29). Many studies have shown that GDNF protects motoneurons from degeneration in vitro $(30,31)$. BDNF promotes the survival of developing motoneurons in vitro and rescues motoneurons from axotomy-induced cell death in vivo (32). In adult rodents, administration of BDNF and GDNF has been reported to prevent the loss of spinal motoneurons after spinal root avulsion $(33,34)$. In vitro studies have demonstrated that motoneurons can respond to NT3 by exhibiting prolonged survival (35). TGF- $\alpha$ is a factor known to support the survival of motoneurons (36) and axonal regrowth after axotomy (37). Human embryonic germ cell derivatives facilitate motor recovery of rats with diffuse motoneuron injury via enhancement of host neuron survival by secreting BDNF and TGF- $\alpha$ (38).

VEGF is known to exert a proangiogenic effect $(39,40)$. Angiogenesis factors are crucial for shaping the nervous system and protecting it from disease. These factors are now known to have crucial roles in neurogenesis, neuroprotection, and the pathogenesis of MND and other neurological diseases (41). Recently, VEGF has been identified as neuroprotective for motoneurons and has been implicated in the pathogenesis of ALS (42). 
The chemokine KC (CXCL1) is highly expressed in the developing brain and acts specifically through CXCR2 receptors in neurons, oligodendrocytes, and astrocytes in both rodents and humans (43, 44). CXCL1 and CXCR2 receptors in the human fetal brain are preferentially localized to cortical ventricular/subventricular areas where neural stem/progenitor cells are also located (44). G-CSF is an essential growth factor in hematopoiesis and may play a role in brain repair. It is neuroprotective and beneficial to functional restoration when administered after brain ischemia (45) and may also have significant neuroprotective effects on motoneuron cell lines in models of ALS (46). A pilot study of G-CSF mobilization of peripheral blood stem cells in ALS has been conducted, under the hypothesis that transiently increasing the number of circulating hematopoietic stem cells might be beneficial in treatment of this disease (47). Primed ALDH ${ }^{\text {hiSSC }}{ }^{\text {lo }}$ NSCs could also share a mechanism of action with genetically engineered cells to express neurotrophic factors $(31,48,49)$. Overall, the ability of $\mathrm{ALDH}^{\text {hisSClo }}$ primed NSC cells to migrate from CSF, secrete trophic factors, and generate motoneurons makes these cells particularly promising for cell therapeutic approaches.

In conclusion, we have demonstrated that transplantation with an NSC subpopulation has beneficial effects on SMA mice, suggesting that this class of stem cell may have a role in the development of MND therapies, by both neurogenesis and the induction of neuroprotective mechanisms. It is conceivable that in the future, stem cell transplantation could be combined with other molecular and pharmacologic approaches to achieve an effective recovery.

\section{Methods}

Animal models. This triple mutant SMA mouse harbors 2 transgenic alleles and a single targeted mutant. $\mathrm{The} \operatorname{Tg}(\mathrm{SMN} 2 *$ delta7) $4299 \mathrm{Abmb}$ allele consists of an SMN cDNA lacking exon 7 (SMN $\Delta 7$ ), whereas the $T g(S M N 2) 89 A b m b$ allele consists of the entire human SMN2 gene.

Heterozygous $S m n$-knockout mice with human SMN2 transgenes were crossed to generate transgenic mice that were homozygous for the knock-

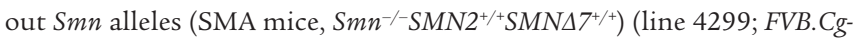
$\mathrm{Tg}\left(\mathrm{SMN} 2 *\right.$ delta7) $4299 \mathrm{Ahmb} \mathrm{Tg}(\mathrm{SMN} 2) 89 \mathrm{Ahmb}$ Smn $\left.1^{\text {tm } 1 \text { Msd }}\right)$. The mice were genotyped using a PCR-based assay on genomic DNA from tail biopsies, as described previously (4).

As the cell donor, we used mHB9-GFP1b transgenic mice, expressing eGFP DNA under the control of the mouse HB9 promoter in the cell bodies of spinal motoneurons in E9.5-P10 mice, enabling immediate detection of the acquired motoneuronal phenotype (10).

All transgenic animals were purchased from The Jackson Laboratory. All animal experiments were approved by University of Milan and Italian Ministry of Health review boards, in compliance with NIH guidelines (9).

Isolation and culture of NSCs. NSCs were isolated from the spinal cord of HB9-GFP mice at E12.5 as described previously (9). Isolated spinal cords were mechanically dissociated with a Pasteur pipette and incubated in $0.05 \%$ trypsin/EDTA solution for 15 minutes at $37^{\circ} \mathrm{C}$. Single-cell suspension was seeded at a density of 100,000 cells $/ \mathrm{ml}$ in Neurobasal medium (GIBCO; Invitrogen), containing B-27, N2 (Invitrogen), EGF (20 ng/ml; Sigma-Aldrich), FGF (20 ng/ml; Sigma-Aldrich), and penicillin (100 U)/ streptomycin $(100 \mu \mathrm{g} / \mathrm{ml} / \mathrm{ml}$; Invitrogen). Cells were grown in uncoated T75 plastic flasks (NUNC; Nalgene Nunc International Corp.) as freefloating clusters (neurospheres). The cultures were passaged every 5-7 days. Cells used for separation were passaged 3-5 times.

Selection of ALDH ${ }^{\text {hi }} \mathrm{SSC}^{\text {lo }}$ cells. ALDH ${ }^{\text {hiSSClo }}{ }^{\text {lo }}$ cells were isolated from spinal cord neurospheres as previously described (9). For ALDH staining, the cells were suspended in ALDEFLUOR assay buffer containing ALDH sub- strate, BAAA, for 60 minutes, following the manufacturer's instructions (StemCell Technologies Inc.). After staining, cells were kept in ice during all subsequent procedures. In each experiment, a sample of cells was stained under identical conditions with a specific ALDH inhibitor, diethylaminobenzaldehyde (DEAB), as a negative control, following the manufacturer's instructions. Flow cytometric sorting was performed using a FACS Vantage SE (BD - Immunocytometry System). ALDEFLUOR fluorescence was excited at $488 \mathrm{~nm}$, and fluorescence emission was detected using a standard 530/30 band-pass filter. Low side scatter (SSC ${ }^{\text {lo }}$ ) and a high ALDH $\left(\mathrm{ALDH}^{\mathrm{hi}}\right)$ cell subset were selected.

Cell culture and differentiation of $A L D H^{\text {hi }} S S C^{l o}$ cells. For cell expansion and clonal culture, $\mathrm{ALDH}^{\text {hiSSClo }}{ }^{\text {lo }}$ sorted cells were plated in a previously described growth medium (Neuroepithelial medium) containing FGF and EGF (50). For in vitro priming (51), NSCs were cultured as previously described (8) in Neurobasal plus N2, $0.1 \mathrm{mM}$ 2-mercaptoethanol, $20 \mathrm{ng} / \mathrm{ml}$ bFGF, $1 \mu \mathrm{g} / \mathrm{ml}$ laminin, $5 \mu \mathrm{g} / \mathrm{ml}$ heparin, $10 \mathrm{ng} / \mathrm{ml}$ neural growth factor (NGF) (Invitrogen), $10 \mathrm{ng} / \mathrm{ml} \mathrm{Shh} \mathrm{(R \& D} \mathrm{Systems),} 10 \mu \mathrm{M}$ forskolin (Sigma-Aldrich), and RA (1 $\mu \mathrm{M})$ (Sigma-Aldrich) for 48 hours or 5 days. After that, GDNF, BDNF, ciliary neurotrophic factor (CNTF), IGF, and NT3 (10 ng/ml; R\&D Systems) were added to the medium.

Immunocytochemistry on cell culture. Cultured cells were fixed in 4\% PFA (10 minutes) at room temperature. After rinses with PBS and preincubation in a mixture of $5 \%$ normal serum and $0.25 \%$ Triton X-100 in PBS, the cultures were incubated with the primary antibodies (see below) overnight at $4^{\circ} \mathrm{C}$. The following proteins were evaluated: beta III-tubulin (TuJ1) (mouse monoclonal, 1:200; Chemicon International), phosphorylated NF-M and NF-H (mouse monoclonal, 1:200; Chemicon International), anti-MAP2 (mouse monoclonal, 1:100; Sigma-Aldrich), anti-ChAT (rabbit, 1:100; Chemicon International), anti-islet-1 (rabbit, 1:200; Chemicon International), 27 anti-HB9 (rabbit, 1:200; Chemicon International), and Alexa Fluor 488 antibodies recognizing GFP (rabbit polyclonal, 1:400; Molecular Probes, Invitrogen); rhodamine-conjugated bungarotoxin was purchased from Molecular Probes, Invitrogen (1:1,000).

After repeated rinses in PBS, the primary unconjugated antibodies were further incubated with FITC and R-Phycoerythrin (RPE) or TRITC-conjugated secondary antibodies (1:100; DAKO) (1 hour, dark, room temperature) in PBS, then rinsed in PBS and coverslipped. Controls with omission of primary antibodies were made, with no detection of positive signals.

Ten monolayer fields (more than 200 cells) were randomly chosen for each sample for quantitative analyses of cell phenotypes of ALDH ${ }^{\text {hiSSC }}{ }^{\text {lo }}$ differentiated in vitro. The percentage of any given phenotype in a sample was obtained by averaging proportions of a specific cell type in each of the 10 fields. At least 4 samples were counted for each treatment group.

$A L D H^{\text {biSSClo }}$ cell transplantation. Before cell transplantation, ALDH ${ }^{\text {hiSSClo }}$ cells from HB9-GFP mice were cultured (for priming) and harvested as previously described (8). We also transplanted ALDH ${ }^{\text {hiSSClo }}$ cells in undifferentiated

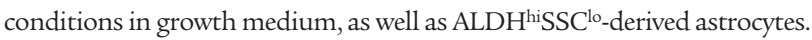

We prepared glial monolayers differentiating NSCs in NeuroCult Differentiation medium (StemCell Technologies Inc.) for 1 week, then harvested the cells with $0.25 \%$ trypsin (Invitrogen) and plated them onto glass supports as previously described (52). After 2 weeks, glial cultures contained $95 \% \mathrm{GFAP}^{+}$astrocytes and no neurons or oligodendrocytes. These cells were then harvested for transplantation.

Murine dermal fibroblasts (primary culture from neonatal skin) were cultured as controls, as described previously (53). Undifferentiated cells, astrocytes, and fibroblasts were prepared for transplantation identically to the "primed" cells (8).

One-day-old SMA mouse pups were used as graft recipients. Furthermore, to easily evaluate cell distribution over a short time (1-2 days and 5-7 days after transplantation), donor cells were labeled with fluorescent dye PKH26 
(Sigma-Aldrich) following the manufacturer's instructions. One-day-old SMA mouse pups were used as graft recipients. Cells were transplanted into the CSF of cryoanesthetized animals as previously described $(9,54)$. To ensure correct transplantation into the CSF, we injected the cells with a solution of dye tracker (lissamine green). The appearance of the marker dye along the spinal cord and in the fontanelles, visible through the skin, indicated the successful delivery of the cells into the spinal canal (55). A total of $2 \mu \mathrm{l}$ of cell suspension ( 20,000 cells) was slowly injected. As controls, SMA and WT mice were injected with vehicle by the same surgical procedure.

SMA mice were divided into treated (transplantation with ALDH ${ }^{\text {hiSSClo }}$ "primed" cells; $n=24: 12$ males) and untreated (vehicle-injected; $n=24: 12$ males) groups that were evaluated up to the end stage for neuromuscular evaluation, survival record, and histological evaluation of donor cell phenotype. The study was designed so that littermates were distributed equally into the treated and untreated groups. Furthermore, 3 groups of animals (each composed of 6 mice/time point for both treated SMA and WT mice) were sacrificed at 5,7 , and 10 days of age for migration analysis. Another 3 groups (treated and untreated SMA and WT mice) were analyzed for histological quantification (for each group/point: $n=6 ; 10-13$ days) and for molecular analysis (for each group/time point: $n=6 ; 13$ days). Finally, the survival curve was plotted for the animal group transplanted with $\mathrm{ALDH}^{\text {hi }}$ SSC $^{\text {lo }}$ undifferentiated cells ( $n=24: 12$ males), ALDH $^{\text {hiSSC }}{ }^{\text {lo }}$-derived astrocytes ( $n=24: 12$ males), and primary fibroblasts ( $n=24: 12$ males).

Assessment of survival. Treated and untreated SMA mice were monitored daily following transplantation for clinical signs of disease. The investigators performing the functional assessment were blind to the treatment. The mice were killed at the clinical end point when they presented difficulties in feeding, a clear downward trend (mice with $30 \%$ weight loss and severe paralysis), and breathing problems, as previously described (18). Body weight was recorded daily.

Assay of strength in SMA mice. SMA mice were tested to evaluate grip strength as previously described (11). Observers were blinded to all groups during the tests. Control mice as well as treated and untreated SMA mice between 10 and 15 days of age were timed to see how long they could support their weight holding onto a metal rail suspended in midair. Each mouse was subjected to 5 trials with at least a 10 -minute rest period between tests. The data were analyzed by ANOVA followed by a Tukey posthoc analysis for multiple comparisons.

Open-field test. Ambulatory behavior was assessed in an open-field test (11). The apparatus consisted of a wooden box measuring $28 \times 28 \times 5 \mathrm{~cm}$. The floor was divided into 16 equal squares of $7 \times 7 \mathrm{~cm}$. The mice were tested individually, and the open field was washed after each session. Each mouse was placed in the center of the open field. It was allowed to move freely for 5 minutes, and data were scored manually by the observer blind to the groups.

Tissue analysis. The animals were sacrificed, perfused, and fixed with $4 \%$ PFA in PBS (pH 7.4). The spinal cord was isolated, immersed in PFA solution for 1 hour, then in sucrose $20 \%$ solution in PBS ( $\mathrm{pH} 7.4$ ) overnight, and frozen in Tissue-Tek OCT compound with liquid nitrogen. The tissues were cryosectioned and mounted on gelatinized glass slides. Every tenth section of $20 \mu \mathrm{m}$ was collected. All sections were blocked with $1 \%$ FCS in PBS and permeabilized with $0.25 \%$ Triton X-100. Sections were processed for multiple markers to determine the cellular phenotype of GFP-labeled cells. Primary antibodies were added overnight at $4^{\circ} \mathrm{C}$ at dilutions of 1:200 for NeuN (mouse monoclonal antibody; Chemicon International), 1:200 for NF (mouse monoclonal antibody; Chemicon International), 1:200 for TuJ1 (mouse monoclonal antibody; Chemicon International), 1:200 for MAP2 (mouse monoclonal antibody; Sigma-Aldrich), 1:200 for nestin (mouse monoclonal; Chemicon International), 1:100 for anti-ChAT (rabbit; Chemicon International), 1:200 for anti-islet-1 (rabbit; Chemicon
International), and 1:200 for anti-HB9 (rabbit; Chemicon International). Donkey-goat, mouse, rabbit, or rat antibodies conjugated with FITC, RPE, CY3, or biotin (1:200; Jackson ImmunoResearch Laboratories Inc. and Dako) were used for 1 hour at room temperature as secondary antibodies when unconjugated primary antibodies were used.

Immunohistochemistry for GFAP, O4, and nestin with FISH was performed as described previously (9). Anti-GFP antibody rabbit serum Alexa Fluor 488 (1:400 dilution; Molecular Probes, Invitrogen) was used to reveal GFP positivity.

Coexpression of GFP and tissue-specific markers was evaluated by conventional fluorescence microscope (Zeiss Axiophot) and by laser confocal scanning (Leica TCS SP2 AOBS) microscopic analysis.

Optical dissectors and random sampling were used to obtain an unbiased stereological estimation of GFP-positive cells and FISH Y-positive donor cells. For donor cell quantification, a systematic random series of every tenth coronal section $(20 \mu \mathrm{m})$ was obtained throughout the entire spinal cord (a mean of 25 sections per animal).

Numerical cell density was then estimated using the optical dissector method $(56,57)$. Optical dissectors sized $100 \times 70 \times 14 \mu \mathrm{m}$ were randomly sampled, and the number of positive cells in each dissector was quantified. The density was calculated by dividing the total number of donor cells by the total volume of optical dissectors. The total volume of tissue per specimen (Vcord) containing donor cells was calculated using the Cavalieri method. This total volume of tissue, multiplied by the number of donor cells per $\mu \mathrm{m}^{3}(\mathrm{Nv})$, gave the total number of donor cells per specimen $(n=\mathrm{Nv} \times$ Vcord) $(57)$.

Histological analysis and counting motoneurons. The lumbar spinal cord region was processed for paraffin embedding. Serial cross-sections ( $12 \mu \mathrm{m}$ thickness) of the lumbar spinal cords were made, among which 1 of every 5 sections was processed and Nissl stained, as reported previously (11). The number and diameter of all motoneurons counted in these cross sections ( $n=50$ for each mouse) were analyzed. The sections were analyzed at an $\times 200$ magnification in the anterior horn (either left or right) for the presence of all neurons in that region. All cells were counted within the ventral horn below an arbitrary horizontal line drawn from the central canal. Only neuronal cells showing at least 1 nucleolus located within the nucleus were counted, as previously described (11).

Histological analysis of muscles. Distal hind limbs were dissected and frozen in Tissue-Tek OCT compound with liquid nitrogen. The tissues were crosssectioned at the midpoint of the muscle. Sections $(10 \mu \mathrm{m})$ were mounted on slides and stained with H\&E (50 sections for each animal). Digital images were captured using a Zeiss Axioscope microscope and analyzed with NIH ImageJ software (http://rsbweb.nih.gov/ij/) for total TA cross-sectional area (original magnification, $\times 5$ ), total TA myofiber number (original magnification, $\times 10$ ), and myofiber diameter (original magnification, $\times 40$ ). Myofiber diameter was determined by measuring the largest diameter of at least 300 neighboring myofibers per animal.

For NMJ staining, 30- $\mu \mathrm{m}$ longitudinal sections were cut on a cryostat and mounted onto gelatin-coated slides. The sections were incubated for 3 hours at room temperature with rhodamine-conjugated $\alpha$-bungarotoxin (50 ng/ml; Molecular Probes, Invitrogen) dissolved in PBS. The slides were rinsed and mounted. A total of $70 \mathrm{NMJs}$ for each mouse were evaluated. The slides were observed through a confocal microscope (Leica) and analyzed with NIH ImageJ software.

LCM of motoneurons. Motoneurons from the lumbar spinal tract of treated SMA mice $(n=3)$, untreated SMA mice $(n=3)$, and WT mice $(n=3)$ were obtained by LCM and analyzed. The latter groups underwent the surgical procedure with vehicle. The surgical procedure and cell transplantation were done at P1, and the animals were sacrificed at 13 days. Spinal cords (lumbar regions L1-L5) of mice perfused with PFA were rapidly removed, 
embedded in Tissue-Tek OCT compound, frozen in liquid nitrogen, and stored at $-80^{\circ} \mathrm{C}$. Tissues were sectioned at $16 \mu \mathrm{m}$ and mounted on covered membrane slides (Leica). The sections were stored at $-80^{\circ} \mathrm{C}$ until microdissection. The sections were immunostained for ChAT (goat polyclonal, 1:200; Chemicon International) for 1 hour. After primary antibody incubation, secondary antibody (1:200; Jackson ImmunoResearch Laboratories Inc.) followed for 30 minutes.

Motoneurons were microdissected using the Leica Laser Microdissection Microscope-ASLMD (Leica). Criteria for endogenous motoneuron selection included: (a) localization in the ventral part of the spinal cord; (b) a diameter of at least $25 \mu \mathrm{m}$; (c) an identifiable nucleus; and (d) positivity for ChAT. ChAT-positive cells that were also positive for HB9-GFP were excluded because they were of donor origin.

Dissected motoneurons were catapulted into a microfuge cap moistened with a drop of mineral oil (Sigma-Aldrich). Approximately 100 cells were collected per cap. A total of 900-1,200 motoneurons were used for analysis on 1 GeneChip array and for quantitative real-time PCR analysis. Motoneurons were pooled from 1 animal per GeneChip. Additionally, we collected 900-1,200 motoneurons to confirm microarray results by quantitative real-time PCR.

RNA extraction and quality test. Total RNA from the microdissected motoneurons was isolated using an RNeasy Micro Kit (QIAGEN) including DNase treatment to remove potential genomic DNA contamination. The starting RNA (100 ng) was quantified by spectrophotometric analysis (ND-100; NanoDrop, Thermo Scientific). Its quality was satisfactory and suitable for the following double amplification process. RNA quality was assessed after both amplifications using spectrophotometric and electrophoretic analysis on agarose gel.

cRNA preparation, oligonucleotide microarray bybridization, and analysis. Hybridization targets were obtained following a double amplification procedure according to the protocol developed by Affymetrix (GeneChip Eukaryotic Small Sample Target Labeling Assay Version II). A hybridization mixture containing $15 \mu \mathrm{g}$ biotinylated cRNA was generated. The biotinylated cRNA was hybridized to Affymetrix GeneChip Mouse Genome $430 \mathrm{~A}$ arrays at $47^{\circ} \mathrm{C}$ overnight. Three chips, each corresponding to biological triplicates, were hybridized for each condition (WT, treated SMA, and untreated SMA). Chips were visualized on a GeneArray 2500 Scanner (Affymetrix) and image files analyzed.

All experiments were validated after direct measures of cRNA quality: $5^{\prime}: 3^{\prime}$ ratios for $\beta$-actin and for GAPDH (determined from Affymetrix chip hybridization analysis) are indirect indicators of overall RNA preservation. The default parameters were used for the statistical algorithm and for probe set scaling.

Data analysis. All primary microarray data are available at the GEO website: http://www.ncbi.nlm.nih.gov/geo/; GEO accession number: GSE10224.

The microarray data were derived from 3 different groups: WT (vehicletreated), untreated SMA (vehicle-treated), and treated SMA mice. Each population consisted of 3 RNA profiling samples. Our analysis was based on 2 outputs of Affymetrix GeneChip arrays: "signal" (expression level) and "detection" (absent/present/marginal) calls were generated for each gene. Subsequent analyses were performed with Microsoft Excel (Microsoft Office package), R free software (http://www.r-project.org/), and in-house analytical and statistical tools. Only probes with an intergroup homogeneity ( 2 of 3 ) in the detection call were considered for further analysis. Signal expression for treated, untreated, and WT groups were used to compute $\log _{2}$ ratios of untreated versus WT, treated versus untreated, and treated versus WT. Only the probes with a mean of the $\log _{2}$ ratios falling between -1.2 and 1.2 were selected (i.e., with a mean ratio greater than 2.3 or less than 0.43 ) for pairwise analysis. To reduce the variability, the probes with a high SD (greater than the 95th percentile of the distribution) in the $\log _{2}$ ratios were discarded. These selections resulted in a total of 42 elements for WT versus untreated and 31 for treated versus untreated. A hierarchical cluster analysis was computed using the complete method. Every probe was associated with its NCBI identifier, tissue of expression, and gene name to assess a biological trait for each group.

Quantitative real-time PCR. Total RNA from 900-1,200 microdissected motoneurons obtained from 3 animals for each condition (WT and treated and untreated SMA) was extracted as described above. One round of amplification was done following the first cycle (first cDNA and cRNA synthesis) of the Affymetrix double amplification procedure before undertaking reverse transcription into complementary DNA using a Ready-To-Go kit (GE Healthcare). Total RNA was reverse transcribed into complementary DNA using random hexamers and Transcriptor Reverse Transcriptase (Roche Diagnostics). Real-time PCR was performed according to the manufacturer's protocol using TaqMan Gene Expression Assays and an ABI Prism 7000 Sequence Detection System (Applied Biosystems). The gene expression assays used were the following: Socs3 (Mm00545913_s1; suppressor of cytokine signaling 3), Hsbp1 (Mm00834384_g1; heat shock protein 1), Cdkn1a (Mm00432448_m1; cyclin-dependent kinase inhibitor 1A_P21), Dusp1 (Mm01309843_g1; dual specificity phosphatase 1), Anln (Mm00503741_m1; anillin), and Egr1 (Mm00656724_m1; early growth response 1). Primers and probes for SMN full-length and SMN $\Delta 7$ transgenes were designed as previously described (58). The reactions were performed in triplicate and averaged, and SMN Ct values were corrected for 18S (Hs99999901_s1) Ct values using the $\Delta \Delta$ Ct method.

Western blot analysis. Twenty micrograms of protein extract were analyzed from the lumbar spinal cord tract of treated SMA mice, untreated SMA mice, and WT mice at 13 days of age. We also extracted protein extract from primary spinal cord motoneurons cocultured in vitro with NSCs at different times (T0, 48 hours, and 72 hours). The protein extract was separated on a $9 \%$ acrylamide gel and electrophoretically transferred to a nitrocellulose membrane. The blots were probed for expression of SMN, p21, and Hsbp1. Secondary peroxidase-conjugated antibodies were used, and the signal was detected with an ECL detection kit (Amersham).

Coculture of PMNs and NSCs. We derived PMN cultures from E12.5 SMA and WT mice as previously described (59). The culture medium was a motoneuron medium supplemented with a cocktail of trophic factors (59). We performed a coculture assay to separate the PMNs from the NSCs with a microporous membrane.

The PMNs were plated on the bottom of a 24-well (lower) compartment, then we added a microporous membrane (Transwell insert, $0.4 \mu \mathrm{m}$ ) and seeded the NSCs in the internal compartment of the insert (upper compartment). The cells were harvested at different time points for Western blot analysis (T0, 48 hours, and 72 hours) ( $n=4$ for each time point and each condition).

Axon length and growth cone measurement. Axon and growth cone analyses was performed as described previously (13). Motoneurons grown for 7 days on glass coverslips, in coculture assay, were fixed with PFA and subsequently with acetone. The cells were incubated overnight at $4{ }^{\circ} \mathrm{C}$ with primary antibodies as follows: rabbit antibodies against phospho-tau $(1 \mu \mathrm{g} / \mathrm{ml}$; Sigma-Aldrich) and NF-M (1:500; Abcam) and a mouse mAb against MAP2 antibody (1:1,000; Chemicon International). Cells were then washed and incubated for 1 hour at room temperature with conjugated secondary antibodies (1:200; Dako). After washing, the coverslips were mounted.

Only motoneurons that allowed a clear distinction between axons and dendrites were scored for the quantification of neurite length and growth cone area ( $n=3-6$ for each condition). Axons of motoneurons were identified by their length as processes that were at least twice as long as dendrites. Only the longest axonal branches were measured. Cultures obtained from mutant and control embryos from different litters were 
analyzed under a confocal microscope (Leica), and axon length was measured using NIH ImageJ software.

Multiplex cytokine assay. Procarta protein profiling assays (Panomics) were used to simultaneously detect 26 different mouse cytokines per reaction in the cell culture supernatants. This assay uses xMAP technology (multi-analyte profiling Luminex technology) to enable the detection and quantification of multiple protein targets simultaneously. The analyzed cytokines were IL-9, IL-1 $\beta$, IL-2, IL-3, IL-4, IL-5, IL-6, IL-12p40, IL-12p70, IL-17, IL-13, KC, RANTES, IFN- $\gamma$, GM-CSF, TNF- $\alpha$, macrophage inflammatory protein 1- $\alpha$ (MIP1- $\alpha$ ), eotaxin, MCP1, IP10, MCP3, VEGF, IL-23, IL- $1 \alpha$, TGF- $\beta$, and G-CSF. We analyzed primed ALDH ${ }^{\text {hi SSC }}{ }^{\text {lo }}$ and undifferentiated cells, astrocytes, and fibroblasts. The cell culture supernatants were collected after 24 hours $\left(1.5 \times 10^{5}\right.$ cells, $1.5 \mathrm{ml}$ medium; 8 separate experiments for each condition). The samples were processed by Panomics according to the standard operating procedures of the company. VEGF released in supernatants collected from in vitro experiments was also confirmed using ELISA as directed by the manufacturer (R\&D Systems).

ELISA assay. BDNF, GDNF, and NT3 secretion by ALDH ${ }^{\text {hiSSClo-primed }}$ and undifferentiated cells was assessed using commercially available ELISAs, following the manufacturer's instructions (Promega). The TGF- $\alpha$ ELISA was performed as previously described (60). Medium was added $\left(1.5 \times 10^{5}\right.$ cells, $1.5 \mathrm{ml}$ medium $)$ and 24 hours later was removed for ELISA (12 independent experiments for each cytokine). The data were analyzed with Student's $t$ test.

Neutralizing antibodies. Incubation of PMNs and primed ALDH ${ }^{\text {hisSC }}{ }^{\text {lo }}$ cell cocultures was performed with the following neutralizing antibodies: anti-TGF- $\alpha$ antibody (Calbiochem, EMD Biosciences), anti-BDNF (Calbiochem, EMD Biosciences), anti-GDNF (R\&D Systems), anti-NT3 (R\&D Systems), anti-VEGF (R\&D Systems), and control mouse IgG (SigmaAldrich), used at $0.5 \mu \mathrm{g} / \mathrm{ml}$.

Statistics. Kaplan-Meier survival analysis and the log-rank test were used for survival comparisons. The growth curve, assay of strength, and open field were analyzed by ANOVA followed by Tukey post-hoc analysis for multiple comparisons. Numbers and size of motoneurons as well as muscle and NMJ data were statistically evaluated by 1-way ANOVA followed by Tukey post-hoc analysis. The statistical analysis of real-time PCR data was performed with Student's $t$ test. For cytokine detection and for coculture assays, differences between means were analyzed by 2-tailed Student's $t$ test. We used StatsDirect for Windows (version 2.6.4) for all of these statistical analyses. In all analyses, the null hypothesis was rejected when $P$ was less than 0.05 .

\section{Acknowledgments}

The financial support of the following research grants to G.P. Comi and S. Corti is gratefully acknowledged: Italian Ministry PRIN 2007, "Molecular pathogenesis of motoneuron disorders as a tool for the identification of novel biomolecular and cellular therapeutic targets"; Telethon grant GGP06043, "Development of cellular and molecular therapeutic approaches for Spinal Muscular Atrophy with Respiratory Distress (SMARD1)"; AFM 2006, CL/NM 2006.0783/11750, "Development of neural stem cell transplantation as a potential therapy of spinal muscular atrophy"; and a Cariplo Foundation grant. We wish to thank the Associazione Amici del Centro Dino Ferrari for their support. We wish to thank Mirella Meregalli for her technical help and Manuela Sironi for the revision of the manuscript.

Received for publication February 24, 2008, and accepted in revised form July 9, 2008.

Address correspondence to: Giacomo P. Comi, Department of Neurological Sciences, University of Milan, IRCCS Foundation Ospedale Maggiore Policlinico, Mangiagalli and Regina Elena, Padiglione Ponti, Via Francesco Sforza 35, 20122 Milan, Italy. Phone: 390255033817; Fax: 390250320430; E-mail: giacomo. comi@unimi.it.
1. Roberts, D.F., Chavez, J., and Court, S.D. 1970. The genetic component in child mortality. Arch. Dis. Child. 45:33-38.

2. Lefebvre, S., et al. 1995. Identification and characterization of a spinal muscular atrophy-determining gene. Cell. 80:155-165.

3. Lefebvre, S., et al. 1997. Correlation between severity and SMN protein level in spinal muscular atrophy. Nat. Genet. 16:265-269.

4. Le, T.T., et al. 2005. SMNDelta7, the major product of the centromeric survival motor neuron (SMN2) gene, extends survival in mice with spinal muscular atrophy and associates with full-length SMN. Hum. Mol. Genet. 14:845-857.

5. Flax, J.D., et al. 1998. Engraftable human neural stem cells respond to developmental cues, replace neurons, and express foreign genes. Nat. Biotechnol. 16:1033-1039.

6. Park, K.I., Teng, Y.D., and Snyder, E.Y. 2002. The injured brain interacts reciprocally with neural stem cells supported by scaffolds to reconstitute lost tissue. Nat. Biotechnol. 20:1111-1117.

7. Lee, J.P., et al. 2007. Stem cells act through multiple mechanisms to benefit mice with neurodegenerative metabolic disease. Nat. Med. 13:439-447.

8. Corti, S., et al. 2007. Neural stem cells LewisX+ CXCR4+ modify disease progression in an amyotrophic lateral sclerosis model. Brain. 130:1289-1305.

9. Corti, S., et al. 2006. Transplanted ALDHhiSSClo neural stem cells generate motor neurons and delay disease progression of nmd mice, an animal model of SMARD1. Hum. Mol. Genet. 15:167-187.

10. Wichterle, H., Lieberam, I., Porter, J.A., and Jessell, T.M. 2002. Directed differentiation of embryonic stem cells into motor neurons. Cell. 110:385-397.

11. Grondard, C., et al. 2005. Regular exercise prolongs survival in a type 2 spinal muscular atrophy model mouse. J. Neurosci. 25:7615-7622.

12. Nicole, S., et al. 2003. Intact satellite cells lead to remarkable protection against Smn gene defect in differentiated skeletal muscle. J. Cell Biol. 161:571-582.

13. Rossoll, W., et al. 2003. Smn, the spinal muscular atrophy-determining gene product, modulates axon growth and localization of beta-actin mRNA in growth cones of motoneurons. J. Cell Biol. 163:801-812.

14. Harper, J.M., et al. 2004. Axonal growth of embryonic stem cell-derived motoneurons in vitro and in motoneuron-injured adult rats. Proc. Natl. Acad. Sci. U. S. A. 101:7123-7128.

15. Deshpande, D.M., et al. 2006. Recovery from paralysis in adult rats using embryonic stem cells. Ann. Neurol. 60:32-44.

16. Shihabuddin, L.S., Horner, P.J., Ray, J., and Gage, F.H. 2000. Adult spinal cord stem cells generate neurons after transplantation in the adult dentate gyrus. J. Neurosci. 20:8727-8735.

17. Lesbordes, J.C., et al. 2003. Therapeutic benefits of cardiotrophin-1 gene transfer in a mouse model of spinal muscular atrophy. Hum. Mol. Genet. 12:1233-1239.

18. Azzouz, M., et al. 2004. Lentivector-mediated SMN replacement in a mouse model of spinal muscular atrophy. J. Clin. Invest. 114:1726-1731.

19. Lefebvre, S., Bürglen, L., Frézal, J., Munnich, A., and Melki, J. 1998. The role of the SMN gene in proximal spinal muscular atrophy. Hum. Mol. Genet.
7:1531-1536.

20. Olaso, R., et al. 2006. Activation of RNA metabolism-related genes in mouse but not human tissues deficient in SMN. Physiol. Genomics. 24:97-104.

21. Zhang, Z., et al. 2008. SMN deficiency causes tissue-specific perturbations in the repertoire of snRNAs and widespread defects in splicing. Cell. 133:585-600.

22. Tadesse, H., Deschênes-Furry, J., Boisvenue, S., and Côté, J. 2008. KH-Type splicing regulatory protein interacts with survival motor neuron protein and is misregulated in spinal muscular atrophy. Hum. Mol. Genet. 17:506-524.

23. Fidzianska, A., Goebel, H.H., and Warlo, I. 1990. Acute infantile spinal muscular atrophy: muscle apoptosis as a proposed pathogenetic mechanism. Brain. 113:433-445.

24. Soubrouillard, C., et al. 1995. Expression of developmentally regulated cytoskeleton and cell surface proteins in childhood spinal muscular atrophies. J. Neurol. Sci. 133:155-163.

25. Haslbeck, M., Franzmann, T., Weinfurtner, D., and Buchner, J. 2005. Some like it hot: the structure and function of small heat-shock proteins. Nat. Struct. Mol. Biol. 12:842-846.

26. Fukada, Y., et al. 2007. Gene expression analysis of the murine model of amyotrophic lateral sclerosis: studies of the Leu126delTT mutation in SOD1. Brain Res. 1160:1-10.

27. Evgrafov, O.V., et al. 2004. Mutant small heat-shock protein 27 causes axonal Charcot-Marie-Tooth disease and distal hereditary motor neuropathy. Nat. Genet. 36:602-606.

28. Jablonka, S., Beck, M., Lechner, B.D., Mayer, C., 
and Sendtner, M. 2007. Defective Ca2+ channel clustering in axon terminals disturbs excitability in motoneurons in spinal muscular atrophy. J. Cell Biol. 179:139-149.

29. Lewin, G.R., and Barde, Y.A. 1996. Physiology of the neurotrophins. Annu. Rev. Neurosci. 19:289-317.

30. Henderson, C.E., et al. 1994. GDNF: a potent survival factor for motoneurons present in peripheral nerve and muscle. Science. 266:1062-1064.

31. Suzuki, M., et al. 2007. GDNF secreting human neural progenitor cells protect dying motor neurons, but not their projection to muscle, in a rat model of familial ALS. PLoS ONE. 2:e689.

32. Friedman, W.J., and Greene, L.A. 1999. Neurotrophin signaling via Trks and p75. Exp. Cell Res. 253:131-142.

33. Kishino, A., Ishige, Y., Tatsuno, T., Nakayama, C., and Noguchi, H. 1997. BDNF prevents and reverses adult rat motor neuron degeneration and induces axonal outgrowth. Exp. Neurol. 144:273-286.

34. Li, L., et al. 1995. Rescue of adult mouse motoneurons from injury-induced cell death by glial cell line-derived neurotrophic factor. Proc. Natl. Acad. Sci. U. S. A. 92:9771-9775

35. Henderson, C.E., et al. 1993. Neurotrophins promote motor neuron survival and are present in embryonic limb bud. Nature. 363:266-270.

36. Boillée, S., Cadusseau, J., Coulpier, M., Grannec, G., and Junier, M.P. 2001. Transforming growth factor alpha: a promoter of motoneuron survival of potential biological relevance. J. Neurosci. 21:7079-7088.

37. Herzog, C.D., and Otto, T. 2002. Administration of transforming growth factor-alpha enhances anatomical and behavioral recovery following olfactory nerve transection. Neuroscience. 113:569-580.

38. Kerr, D.A., et al. 2003. Human embryonic germ cell derivatives facilitate motor recovery of rats with diffuse motor neuron injury J. Neurosci. 23:5131-5140.

39. Slevin, M., Kumar, P., Gaffney, J., Kumar, S., and Krupinski, J. 2006. Can angiogenesis be exploited to improve stroke outcome? Mechanisms and ther- apeutic potential. Clin. Sci. 111:171-183.

40. Wei, L.H., et al. 2003. Interleukin-6 promotes cervical tumor growth by VEGF-dependent angiogenesis via a STAT3 pathway. Oncogene. 22:1517-1527.

41. Greenberg, D.A., and Jin, K. 2005. From angiogenesis to neuropathology. Nature. 438:954-959.

42. Bogaert, E., Van Damme, P., Van Den Bosch, L., and Robberecht, W. 2006. Vascular endothelial growth factor in amyotrophic lateral sclerosis and other neurodegenerative diseases. Muscle Nerve. 34:391-405.

43. Robinson, S., and Franic, L.A. 2001. Chemokine GRO1 and the spatial and temporal regulation of oligodendrocyte precursor proliferation. Dev. New rosci. 23:338-345.

44. Filipovic, R., Jakovcevski, I., and Zecevic, N. 2003. GRO-alpha and CXCR2 in the human fetal brain and multiple sclerosis lesions. Dev. Neurosci. 25:279-290.

45. Zhao, L.R., Singhal, S., Duan, W.M., Mehta, J., and Kessler, J.A. 2007. Brain repair by hematopoietic growth factors in a rat model of stroke. Stroke. 38:2584-2591.

46. Tanaka, M., et al. 2006. Intrathecal upregulation of granulocyte colony stimulating factor and its neuroprotective actions on motor neurons in amyotrophic lateral sclerosis. J. Neuropathol. Exp. Neurol. 65:816-825.

47. Cashman, N., et al. 2008. Pilot study of granulocyte colony stimulating factor (G-CSF)-mobilized peripheral blood stem cells in amyotrophic lateral sclerosis (ALS). Muscle Nerve. 37:620-625.

48. Tuszynski, M.H., et al. 2005. A phase 1 clinical trial of nerve growth factor gene therapy for Alzheimer disease. Nat. Med. 11:551-555.

49. Duan, D., Yang, H., Zhang, J., Zhang, J., and Xu, Q. 2005. Long-term restoration of nigrostriatal system function by implanting GDNF genetically modified fibroblasts in a rat model of Parkinson's disease. Exp. Brain Res. 161:316-324

50. Kalyani, A., Hobson, K., and Rao, M.S. 1997. Neu- roepithelial stem cells from the embryonic spinal cord: isolation, characterization, and clonal analysis. Dev. Biol. 186:202-223.

51. Wu, P., et al. 2002. Region-specific generation of cholinergic neurons from fetal human neural stem cells grafted in adult rat. Nat. Neurosci. 5:1271-1278.

52. Silva, G.A., Feeney, C., Mills, L.R., and Theriault, E. 1998. A novel and rapid method for culturing pure rat spinal cord astrocytes on untreated glass. J. Neurosci. Methods. 80:75-79.

53. Huard, C., et al. 1998. Transplantation of dermal fibroblasts expressing MyoD1 in mouse muscles. Biochem. Biophys. Res. Commun. 248:648-654.

54. Elliger, S.S., Elliger, C.A., Aguilar, C.P., Raju, N.R., and Watson, G.L. 1999. Elimination of lysosomal storage in brains of MPS VII mice treated by intrathecal administration of an adeno-associated virus vector. Gene Ther. 6:1175-1178.

55. Fedorova, E., Battini, L., Prakash-Cheng, A., Marras, D., and Gusella, G.L. 2006. Lentiviral gene delivery to CNS by spinal intrathecal administration to neonatal mice. J. Gene Med. 8:414-424.

56. Gundersen, H.J., et al. 1988. The new stereological tools: dissector, fractionator, nucleator and point sampled intercepts and their use in pathological research and diagnosis. APMIS. 96:857-881.

57. Messina, A., Sangster, C.L., Morrison, W.A., and Galea, M.P. 2000. Requirements for obtaining unbiased estimates of neuronal numbers in frozen sections. J. Neurosci. Methods. 97:133-137.

58. Sumner, C.J., et al. 2006. SMN mRNA and protein levels in peripheral blood: biomarkers for SMA clinical trials. Neurology. 66:1067-1073.

59. Locatelli, F., et al. 2007. Fas small interfering RNA reduces motoneuron death in amyotrophic lateral sclerosis mice. Ann. Neurol. 62:81-92.

60. Chen, X., et al. 2007. Integrin alpha1beta1 controls reactive oxygen species synthesis by negatively regulating epidermal growth factor receptor-mediated Rac activation. Mol. Cell. Biol. 27:3313-3126. 